This is an Accepted Manuscript of an article published by Taylor \& Francis in Multivariate Behavioral Research on 17 Oct 2011, available online:

https://www.tandfonline.com/doi/abs/10.1080/00273171.2011.606748 
COMPARISON OF METHODS FOR COLLECTING AND MODELING DISSIMILARITIES 1

Running head:COMPARISON OF METHODS FOR COLLECTING AND MODELING DISSIMILARITIES

Comparison of methods for collecting and modeling dissimilarity data: applications to complex sound stimuli

Bruno L. Giordano, Catherine Guastavino, Emma Murphy, Mattson Ogg, Bennett K. Smith and Stephen McAdams McGill University 
COMPARISON OF METHODS FOR COLLECTING AND MODELING DISSIMILARITIES 2

\author{
Abstract \\ Sorting procedures are frequently adopted as an alternative to dissimilarity ratings to \\ measure the dissimilarity of large sets of stimuli in a comparatively short time. However, \\ systematic empirical research on the consequences of this experiment-design choice is \\ lacking. We carried out a behavioral experiment to assess the extent to which sorting \\ procedures compare to dissimilarity ratings in terms of efficiency, reliability and accuracy, \\ and the extent to which data from different data-collection methods are redundant and \\ are better fit by different distance models. Participants estimated the dissimilarity of \\ either semantically charged environmental sounds or of semantically neutral synthetic \\ sounds. We considered free and hierarchical sorting and derived indications concerning the \\ properties of constrained and truncated hierarchical sorting methods from hierarchical \\ sorting data. Results show that the higher efficiency of sorting methods comes at a considerable \\ cost in terms of data reliability and accuracy. This loss appears to be minimized with truncated \\ hierarchical sorting methods that start from a relatively low number of groups of stimuli. Finally, \\ variations in data-collection method differentially \\ affect the fit of various distance models at both the group-average and individual levels. \\ Based on these results we suggest adopting sorting as an alternative to dissimilarity-rating \\ methods only when strictly necessary. We also suggest analyzing the raw behavioral \\ dissimilarities, and avoiding modeling them with one single distance model. \\ Keywords: Similarity, hierarchical sorting, multidimensional scaling, additive trees, \\ common and distinctive features.
}


COMPARISON OF METHODS FOR COLLECTING AND MODELING DISSIMILARITIES 3

\section{Comparison of methods for collecting and modeling dissimilarity data: applications to complex sound stimuli}

Similarity is a fundamental construct in the empirical and theoretical study of a variety of cognitive and perceptual processes such as categorization, problem solving, generalization and memory retrieval (Tversky, 1977; Shepard, 1987; Goldstone, 1999). Various distance models are available to model dissimilarities as a function of the features of the judged stimuli (Tversky, 1977; see Tversky \& Gati, 1978; Gati \& Tversky, 1982; for differences between similarity and dissimilarity judgments), and empirical studies often aim to measure the features underlying the mental representation of the stimuli (see Borg \& Groenen, 1997, for exploratory and confirmatory approaches). Dissimilarities can be collected with various methods (e.g., Rao \& Katz, 1971; Henry \& Stumpf, 1975; Tsogo, Masson, \& Bardot, 2000). Although several studies have quantified the merits and disadvantages of the various data-collection methods, none has jointly considered all the factors relevant to their comparison: their efficiency, the reliability and accuracy of the data, the similarity of data collected with different methods (redundancy), and the effects of method on the fit of distance models. As a consequence, the methods-comparison literature is widely scattered, and several of these aspects remain partially investigated, at best. We investigated the extent to which the above-mentioned factors vary across data-collection methods. We considered the methods of dissimilarity ratings, hierarchical sorting and free sorting, and modeled the properties of the constrained and truncated hierarchical sorting methods from the hierarchical sorting data.

Among the various methods, that of dissimilarity ratings or paired comparisons is perhaps the most popular. Accordingly, participants rate along a categorical or continuous scale the dissimilarity of each of the $N(N-1) / 2$ possible pairs of $N$ stimuli. Despite its popularity, this method is regarded as relatively inefficient because it requires a large 
number of judgments that grows quadratically with the set size (Rosenberg \& Kim, 1975). Further, the inefficiency of this method makes it unsuitable for perceptual domains subject to considerable carry-over and adaptation effects (e.g., tastes and smells; Lawless, Sheng, \& Knoops, 1995). The inefficiency of this method also makes it prohibitive for investigating large sets of stimuli because the required long experimental sessions would result in fatigue and boredom (M. D. Johnson, Lehmann, \& Horne, 1990; Malhotra, 1990; Bijmolt \& Wedel, 1995) and in uncontrolled fluctuations of the response criteria throughout the experimental session. A number of studies investigated more efficient variants of this method that produce incomplete dissimilarity matrices (e.g., Tsogo et al., 2000). Interestingly, an input spatial representation can be accurately recovered through a multidimensional scaling (MDS) analysis of the incomplete dissimilarity matrix, provided that at least two thirds of the data are available (Spence \& Domoney, 1974) or that only dissimilarities of intermediate magnitude are not available (Graef \& Spence, 1979). For these reasons, incomplete designs are of limited value: they rely on the assumption that data can indeed be accurately represented with an MDS model; they require preliminary estimates of the entire dissimilarity matrix necessary to identify dissimilarities of intermediate magnitude; they reduce the experimentation time by only $33 \%$, at best.

Sorting methods are a widely adopted alternative to dissimilarity ratings. With sorting methods, participants create groups of similar stimuli (Coxon, 1999; see Goldstone, 1994, for cognitive theories on the relationship between similarity and categorization). With free sorting (Miller, 1969; Rosenberg \& Kim, 1975), participants are free to decide on how many groups they should create, whereas with constrained sorting the number of groups is fixed by the experimenter. For both these methods, a binary dissimilarity is derived from the co-occurrence of the stimuli within the groups (dissimilarity $=0$ and 1 if two stimuli are in the same group or not, respectively). The variant of hierarchical sorting (hierarchy-construction method, Harloff \& Coxon, 2005, or successive sorting method, 
Bimler \& Kirkland, 1997) is the behavioral analog of the hierarchical clustering scheme (S. C. Johnson, 1967). With agglomerative hierarchical sorting, participants start from a condition in which each of the stimuli is in a different group and, at each subsequent step, merge together the two most similar stimuli or groups of stimuli until all stimuli are merged together. Dissimilarity can be measured as $N$ minus the number of groups into which the stimulus set is partitioned at the moment the two stimuli are first merged (Rao \& Katz, 1971). Variants of this method are available: divisive hierarchical sorting proceeds in the direction opposite to that of agglomerative hierarchical sorting, starting from the one-group condition (Boster, 1986); truncated agglomerative hierarchical sorting starts with a constrained sorting phase (number of groups $<N$, Harbke, 2003) or with a free sorting phase (Bimler, Kirkland, \& Chen, 1998).

The comparative study of dissimilarity ratings and sorting methods has been fragmentary. The choice of a data-collection method should take into account various factors: method efficiency, data reliability (the extent to which results can be replicated either with the same participants or with a different group of participants) and data accuracy (the extent to which data accurately reflect the features of the investigated stimuli); method redundancy (the extent to which different methods yield comparable data); data-modeling biases (the extent to which data from a given method are optimally accounted for by a particular distance model). To date, no study has jointly considered all these factors, thus making the process of selecting a method difficult at best or uninformed at worst. For example, free sorting is often chosen on the grounds that it is a very efficient alternative to dissimilarity ratings (e.g., in Bijmolt \& Wedel, 1995, free sorting is 2.5 times faster than dissimilarity ratings). However, the price of the increased efficiency is seldom considered: for example, free sorts are known to be less accurate than dissimilarity ratings (Subkoviak \& Roecks, 1976). Further, other differences between free sorting and dissimilarity ratings are simply unknown: no study has compared their reliability; 
redundancy studies (Ward, 1977, Bertino \& Lawless, 1993, Bonebright, 1996, Cartier et al., 2006); are often carried out by focusing on MDS models, rather than on raw data (see Harbke, 2003, for an exception), despite the known inaccuracies of the MDS analysis of the binary free-sorting dissimilarities (Kendall, 1975; Goodhill, Simmen, \& Willshaw, 1995; Simmen, 1996) and the vulnerability of the fit of these models to variations in the distributional properties of the input data (Pruzansky, Tversky, \& Carroll, 1982).

The methodological study of hierarchical sorting is even less developed. The best studied aspects are the redundancy and reliability of this method. When compared with dissimilarity ratings, hierarchical sorts are thus reported to be fairly redundant (Bricker \& Pruzansky, 1970; Harbke, 2003 reports a correlation of .60 between group-average truncated hierarchical sorts and dissimilarity ratings), but are also characterized by a larger degree of interindividual differences (Bricker \& Pruzansky, 1970; see Griffiths \& Kalish, 2002, for the effects of the number of participants on the correlation between groupaverage hierarchical sorts). However, empirical data on other properties of this method are lacking. For example, although Bimler and Kirkland (1997) state that hierarchical sorting cannot be used to investigate more than 16 items because of its inefficiency, it is unknown whether this method still represents a more efficient alternative to dissimilarity ratings. Focusing on data accuracy, Bimler and Kirkland (1997) claim that hierarchical sorts provide more information than free sorting (hierarchical sorting dissimilarities can assume a larger number of different values than can binary free sorting dissimilarities). Consistently, Rao and Katz (1971) showed that hierarchical sorting is the most accurate among a variety of sorting methods. Note, however, that Rao and Katz (1971) investigated simulated and not real behavioral data, and accuracy measures were computed from MDS solutions rather than from the raw data. Finally, hierarchical sorting is claimed to be more suitable for the quantification of interindividual differences than free sorting (Lawless et al., 1995) and to require fewer participants than free sorting, but to be 
more demanding (Bimler \& Kirkland, 1997). Notably however, no clear empirical data are available to substantiate either of these claims.

Empirical studies of dissimilarity often base their conclusions not on analyses of the raw dissimilarity data, but on the parameters of a distance model of the raw dissimilarities. Given the importance of this modeling step, the experimenter might be interested in assessing the extent to which model-based conclusions can be replicated by studies based on different data-collection methods and, above all, they might choose the method whose data are accurately accounted for by the distance model of interest. For instance, an experimenter interested in MDS models might choose the method whose data are better accounted by this model. Thus, hierarchical sorts would be a less than optimal choice for MDS-based studies because each individual yields an ultrametric tree (see Appendix) that can be represented perfectly by a Euclidean space with a rather large number of dimensions ( $N-1$, Holman, 1972; see Carroll \& Pruzansky, 1980, for additional considerations), but would likely be a reasonable choice if the modeling interest is in graph-theoretic structures (e.g., additive trees, see Appendix). In addition to these considerations, the experimenter might also be interested in assessing the extent to which modelbased conclusions can be replicated by studies based on different data-collection methods. To our knowledge, no previous empirical work has explored this important dimension of comparison for the data-collection methods.

We carried out a comparative study of dissimilarity ratings, free sorting and agglomerative hierarchical sorting (referred to simply as hierarchical sorting from now on). In order to increase the generality of the results, behavioral dissimilarities were collected for two largely different sound sets: a semantically neutral set of unrecognizable synthetic sounds and a semantically charged set of recognizable living environmental sounds (Giordano, McDonnell, \& McAdams, 2010). Data-collection methods were compared focusing on various factors of potential interest to the experiment-design process: 
efficiency, reliability, redundancy, data modeling and accuracy. Results for each of these aspects are discussed separately at the end of the relevant parts of the Results section. The data-modeling analysis was complemented by a study of the influence of the distributional properties of the data on model fit (Pruzansky et al., 1982). Various analyses considered truncated hierarchical sorting and constrained sorting data derived from the hierarchical sorting data collected with the experimental participants. The validity of the derived data was assessed when analyzing the redundancy of data from different methods. Given their nature, the conclusions reached for derived data should be taken as an indication of what might be expected from an actual experiment based on these methods.

\section{Empirical study}

\section{Methods}

Participants. Participants ( $\mathrm{N}=120$; age: $\mathrm{M}=23$ yrs, STD $=4$ yrs; gender: 75 female, 45 male) were native English speakers and had normal hearing, as assessed with a standard audiometric procedure (ISO, 2004; Martin \& Champlin, 2000).

Stimuli. We selected two sets of 20 stimuli each. The semantic set comprised highly recognizable vocal and nonvocal living environmental sounds (Giordano et al., 2010). The synthetic set comprised harmonic tones equalized in perceived duration and loudness and differing in attack time, spectral centroid and the ratio between the levels of even and odd harmonics (Caclin, McAdams, Smith, \& Winsberg, 2005, Experiment 3). Each of the three variable parameters had the same range of variation as in Caclin et al. (2005) and could assume one of 20 different values, evenly spaced along a psychophysically linear scale. For each stimulus, the level of the synthesis parameters was selected at random and without replacement from the 20 available values. The sounds in the synthetic set were more perceptually more similar to each other than those in the semantic set, and none of them could be associated with a real-world sound-generating event. We selected two 
COMPARISON OF METHODS FOR COLLECTING AND MODELING DISSIMILARITIES 9

ten-stimulus training sets that were different from the experimental sets. The semantic set comprised five living and five nonliving sounds. For the synthetic set, the three synthesis parameters varied within the same range as for the experimental set.

Apparatus. Sound stimuli were stored on the hard disk of a Mac Pro Quad Core Workstation equipped with an M-Audio CO2 optical-to-coaxial S/PDIF converter. Audio signals were amplified with a Grace Design m904 monitor system and presented through Sennheiser HD595 headphones. Participants were seated in a structurally isolated, soundproofed room with a noise-floor rating of PNC20. Sound peak level was 58 dB SPL on average $(\mathrm{STD}=12 \mathrm{~dB})$.

Design and procedure. We adopted a $2 \times 3$ between-subjects design by combining two levels for the sound set factor (semantic vs. synthetic set) with three levels for the data-collection method factor (dissimilarity ratings, and hierarchical or free sorting). Twenty participants were randomly assigned to each of the six cells of the experimental design.

Before estimating the dissimilarities, participants were familiarized with the stimuli by presenting them all twice in sequence in block-randomized order (inter-stimulus-interval, ISI $=100 \mathrm{~ms}$ ). They were instructed to estimate the maximum and minimum within-set dissimilarities while listening to the sounds. The task of estimating the dissimilarities with one of the three investigated methods began after this familiarization phase.

On each trial of the dissimilarity-rating condition, participants were presented with one of the possible $N(N-1) / 2$ pairs of different sounds ( $N=$ number of stimuli), and rated the dissimilarity of the sounds by moving a slider along a scale marked "very similar" and "very different" at the two extremes. The within-pair order was chosen at random on each trial. 
In the first step of the hierarchical sorting condition, participants were presented with $N$ randomly numbered on-screen icons corresponding to the $N$ sounds. Icons could be dragged around the screen by using the mouse. Participants were asked to listen to each of the sounds by clicking on the icons and to drop the two most similar sounds inside a merging box. When the merging box contained two sounds, participants clicked on an on-screen button labeled "OK" to create a new icon that pointed to the two grouped sounds. The new icon was labeled with the numbers of the icons for the merged stimuli (e.g., "3-6" for the merged icons "3" and "6"). When the participant clicked on an icon for merged sounds, all of the sounds were played back in random order (ISI $=100 \mathrm{~ms}$ ). At each subsequent step of the procedure, participants were asked to drop the two most similar sounds/groups of sounds inside the merging box. Participants were required to listen to each of the stimuli at least once before each of the first three merging decisions. The procedure ended when only two groups of stimuli remained to be merged.

In the free sorting condition, participants were presented with $N$ randomly numbered on-screen icons, one for each of the $N$ stimuli. They were asked to create as many nonempty groups of similar sounds as they thought necessary, but not less than 2 groups and not more than $N-1$. Sounds were grouped by dropping the icons inside a merging box, one for each of the groups. Participants were required to listen to each of the sounds at least twice before creating any group and to listen to each of the groups at least once after each of the sounds had been dropped inside one of the merging boxes.

In all conditions, participants could listen to the stimuli as many times as needed before giving a response. At the beginning of the hierarchical and free sorting tasks, participants were required to arrange the on-screen icons so that similar sounds were closer together. Participants were told that this initial step was meant to aid the process of creating groups of sounds and were instructed to start grouping the sounds that they had arranged closer on the screen. In all conditions, response-related operations (e.g., drag 
the icons or move the slider) were also possible during the playback of the sounds. For all of the conditions, the task was initially practiced with the training set. For the training phase (average duration $=4.2$ minutes; $\mathrm{STD}=4.2$ ), participants rated the dissimilarity of 10 pairs randomly selected out of the possible 45 , or carried out the above-described sorting procedures in their entirety.

Results

All analyses considered group-average data. Individual data were considered in the data-modeling and accuracy analyses. The minimum and maximum possible dissimilarity ratings were 0 and 1 , respectively. With hierarchical sorting, the dissimilarity of two stimuli was computed as $1-N g / N$ [range: $1 / N$ to $(N-1) / N$ ], where $N g$ is the number of groups into which the stimulus set is partitioned at the moment the two stimuli are first merged (Rao \& Katz, 1971). With free sorting, the (binary) dissimilarity of two stimuli equals 0 if the stimuli are grouped together and 1 if they are not. For each of the hierarchical sorting steps, differing in the number of groups of stimuli, we finally computed a binary dissimilarity following the same co-occurrence approach as for the free sorting method. These distance matrices derived from the hierarchical sorting data are taken as an approximation of real constrained sorting dissimilarities (see redundancy analyses for validation). For all methods, group-average dissimilarities were given by the average of individual data ${ }^{1}$.

Efficiency. Table 1 reports four different temporal measures for each of the experimental conditions: experiment duration, playback time, nonplayback time dedicated exclusively to response operations (Tresp), and number of playbacks/stimulus (Nplays). None of these measures considered the initial phase of familiarization with the stimuli. As shown in Table 1, the experiment took more time for the semantic than for the synthetic set. This difference in part reflects a longer average duration of the semantic sounds 
compared to the synthetic sounds, $2.4 \mathrm{~s}(\mathrm{STD}=1.24)$ and $0.6 \mathrm{~s}(\mathrm{STD}=0.01)$, respectively. Nplays was higher for dissimilarity ratings than for hierarchical and free sorting. This difference in part reflects the minimum number of playbacks required for each condition: 19 for dissimilarity ratings and 3 for hierarchical and free sorting. Within two separate $2 \times 3$ ANOVAs, we analyzed the influence of sound set and data-collection method on both Tresp and Nplays. The interaction between sound set and data-collection method was not significant for either temporal factor, $\mathrm{F}(2,114)=2.10$ and $.51, p=.13$ and $.60, \eta_{p}^{2}=.04$ and .01 for Tresp and Nplays, respectively. Data-collection method significantly influenced both variables, $\mathrm{F}(2,114)=5.91$ and $25.17, p=.004$ and $<$ $.001, \eta_{p}^{2}=.09$ and .31 for Tresp and Nplays, respectively. Both variables were higher with dissimilarity ratings than with both sorting methods: for Tresp as dependent variable, unpaired $\mathrm{t}(78)=3.32$ and $2.52, p=.002$ and .01 for hierarchical and free sorting, respectively; for Nplays as dependent variable, unpaired $\mathrm{t}(78)=2.34$ and 5.83 , $p=.02$ and $<.001$ for hierarchical and free sorting, respectively. Whereas Nplays was higher for hierarchical than for free sorting, $\mathrm{t}(78)=4.03, p<.001$, Tresp did not differ significantly between them, $\mathrm{t}(78)=-0.32, p=.75$. Finally, whereas $N$ plays was lower for the semantic than for the synthetic set, $\mathrm{F}(1,114)=35.17, p<.001, \eta_{p}^{2}=.24$, Tresp was not reliably different for the two sound sets, $\mathrm{F}(1,114)=50.89, p=.09, \eta_{p}^{2}=.03$.

\section{[Table 1 here]}

We created a model for predicting the amount of time necessary to evaluate $N$ stimuli with each of the following methods: dissimilarity ratings, free sorting, hierarchical sorting and the truncated hierarchical sorting (see Figure 1). The model extrapolates the empirical efficiency measures obtained with $N=20$ stimuli to various untested stimulus-set sizes. The reader should take the results of this modeling as an indication of the experiment duration that requires a validation through pilot experimental testing. 
Experiment duration was modeled as:

$$
\begin{aligned}
& \text { dissimilarity ratings }\left\{\begin{array}{l}
\text { Tpres }=\text { Tstim } N\left(N-1+k_{1}\right) \\
\text { Tresp }=k_{2} N(N-1) / 2
\end{array}\right. \\
& \text { hierarchical sorting }\left\{\begin{array}{l}
\text { Tpres }=\text { Tstim } N\left(3+k_{1} M\right) \\
\text { Tresp }=k_{2} N M
\end{array}\right. \\
& \text { free sorting }\left\{\begin{array}{l}
\text { Tpres }=\text { Tstim } N\left(3+k_{1}\right) \\
\text { Tresp }=k_{2} N
\end{array}\right.
\end{aligned}
$$

where Tpres is the presentation time for all stimuli; Tstim is the average stimulus-presentation time; $M$ is the number of hierarchical sorting steps $=$ number of starting groups - number of groups after final merge $(M=N-1$ for complete hierarchical sorts that do not omit the last trivial step where all sounds are grouped together; this trivial step is omitted from the simulations; and is not carried out in the behavioral experiments). We considered a minimum Nplays of 3 for hierarchical and free sorting and of $N-1$ for dissimilarity ratings. These values correspond to those used in the actual experiment. The constant $k_{1}$ models the spontaneous Nplays beyond the minimum requirement throughout the entire experiment for dissimilarity ratings and free sorting, and for each of the hierarchical sorting steps. The constant $k_{2}$ models Tresp for each dissimilarity-rating pair or for each stimulus in free sorting or for each stimulus in each of the hierarchical sorting steps. The constants $k_{1}$ and $k_{2}$ were estimated from the empirical data and averaged across sound sets. Predictions were carried out by assuming a stimulus duration of $1 \mathrm{~s}$.

\section{[Figure 1 here]}

Based on this modeling approach, hierarchical and free sorting methods do not appear to be noticeably more efficient than dissimilarity ratings for a number of stimuli lower than 30. For larger sets, free sorting appears instead as an increasingly more 
efficient alternative to both hierarchical sorting and dissimilarity ratings. The comparative gain in efficiency of hierarchical sorting relative to dissimilarity ratings is very small for any set size. Interestingly, truncated hierarchical sorting appears to be highly efficient even when compared with free sorting.

The validity of the efficiency model relies on a number of assumptions. Firstly, for all the hierarchical sorting methods we assume that Tresp and Nplays are constant throughout the merging steps. In practice, participants played the sounds less times and responded faster as they proceeded with the merging task. A more advanced model that takes into account the dependence of Tresp and Nplays on the merging level did not produce substantially different results than those discussed in this section. It is not presented here for the sake of simplicity. Secondly, we assume an average stimulus duration of $1 \mathrm{~s}$. We observed the same pattern of results when assuming a stimulus duration of either $100 \mathrm{~ms}$ or $10 \mathrm{~s}$. Finally, we assumed that each of the following quantities is independent of the number $N$ of stimuli: Tresp $/(N(N-1) / 2)$ for dissimilarity ratings; Tresp $/ N$ for hierarchical and free sorting; $N$ plays for all methods. These last assumptions do not take into account memory limitations. Indeed, it is highly likely that for larger stimulus sets participants will tend to inspect each stimulus a larger number of times than is assumed by our model; and will devote more and more time to response operations simply because they will have a harder time remembering what stimuli they have already inspected, and, for sorting procedures, what stimulus has been placed in which group. For this reason, the estimates of experiment duration are more likely to underestimate the real value as the set size increases and are best conceived as a lower bound that requires a validation through pilot experimental testing.

Reliability. Highly reliable methods yield strongly correlated data with different populations of participants. Based on the assumption that our group of participants is a representative sample of the population, we estimated method reliability by using the 
bootstrap resampling approach (Efron \& Tibishirani, 1993). For each of six target numbers of participants $(x) \log$-spaced from 5 to 160, we computed a bootstrap sample by drawing with replacement two groups of participants of size $x$ from the available data and

then estimating reliability as the $\mathrm{R}^{2}$ between the group-average data for the two sets. The final reliability estimate was the average value across 10,000 bootstrap samples.

Reliability was computed for each of the sound sets, for each of the dissimilarity-rating, and hierarchical and free sorting methods and for the 5-group constrained-sorting data. Although reliability measures were significantly higher for the semantic than for the synthetic set, average $\mathrm{R}^{2}=.83$ and .78 , respectively, paired samples $t(23)=5.99, p<.001$, Cohen's $\mathrm{d}$ for the paired $\mathrm{t}$ test $=.27$, the effect of method on the reliability was highly similar across stimulus sets, $r(22)=.99, p<.001$. This effect will not be discussed further. Figure 2 shows the reliability measures averaged across sound sets.

\section{[Figure 2 here]}

Reliability decreases from dissimilarity ratings to hierarchical sorting to 5-groups constrained sorting to free sorting. The number of participants necessary to reach a target level of reliability increases in the same order. The higher reliability of dissimilarity ratings compared to hierarchical sorts is consistent with the previous observation of larger interindividual differences in hierarchical sorting than in dissimilarity ratings (Bricker \& Pruzansky, 1970). The higher reliability of hierarchical compared to free sorts is consistent with the claim that fewer participants are necessary with the former method (Bimler \& Kirkland, 1997). One likely origin for the effects of method on reliability is the between-methods difference in the number of times participants inspected each of the stimuli: a larger number of inspections of each stimulus indeed allows one to develop a more stable representation and to refine the decision process, thus decreasing the noise in 
the behavioral responses. Consistently, participants listened to each of the sounds more often with dissimilarity ratings than with hierarchical sorting than with free sorting. Another explanation focuses on the resolution of the dissimilarities at the individual level (continuous for dissimilarity ratings, N-1 levels for hierarchical sorting and binary for constrained and free sorting), with higher resolutions allowing responses that more closely reflect the mental dissimilarities. This explanation is less plausible because constrained sorts were more reliable than free sorts in spite of the fact that the individual dissimilarities had the same resolution.

Method redundancy. Redundancy was defined as the proportion of variance $\left(\mathrm{R}^{2}\right)$ shared by group-average data from the different methods. The initial analysis of redundancy also considered the constrained sorts derived from the hierarchical sorting data. The proportion of variance shared between methods was significantly higher for the semantic set than for the synthetic set, average $\mathrm{R}^{2}=.64$ and .63 , respectively, paired samples $t(209)=2.78, p=.006$, Cohen's $\mathrm{d}$ for the paired t test $=.05$. The pattern of between-methods correlations was highly consistent between the two sound sets, $r(208)=.97, p<.001$. Further analyses considered the between-methods $\mathrm{R}^{2}$ matrices averaged across sound sets. We modeled the between-methods distance $1-\mathrm{R}^{2}$ as a minimum variance root additive tree GTREE (Corter, 1998; proportion of explained variance $=.97 ;$ see Figure 3$)$.

\section{[Figure 3 here]}

Hierarchical and free sorts shared a larger proportion of variance, $\mathrm{R}^{2}=.71$, than did any of them with dissimilarity ratings, $\mathrm{R}^{2}$ of dissimilarity ratings with hierarchical or free sorts $=.62$ and .61 , respectively. This result might arise from the fact that the task of creating groups of stimuli is more influenced by categorization processes, whereas that of rating dissimilarities is more influenced by the cognitive estimation of similarities (see 
Goldstone, 1994, for the relationship between similarity and categorization). From a practical point of view, however, this eventual difference in cognitive processes accounts for only $10 \%$ of the data variance. Interestingly, dissimilarity ratings and free and hierarchical sorts are maximally correlated with the constrained sorts derived from the latest steps of the hierarchical merging process (six-group constrained sorts for dissimilarity ratings and free sorting, $\mathrm{R}^{2}=.61$ and .67 , respectively, and seven-group constrained sorts for hierarchical sorting, $\left.\mathrm{R}^{2}=.95\right)$. This similar result might indicate that, independently of whether participants rated dissimilarities or grouped stimuli, they carried out the task by differentiating between very large and smaller mental distances, or by focusing on relatively superordinate levels of their mental taxonomy of the experimental stimuli.

Further, the resemblance of the group-level data to the constrained sorts into 6-7 groups is reminiscent of the number of working-memory chunks (Miller George, 1956), and might thus arise also from limitations in mnemonic resources.

The constrained sorts considered in the redundancy analysis were derived from the hierarchical sorts. We analyzed in detail the redundancy of free and derived constrained sorts in order to assess the extent to which the latter represent an accurate model of what is measured when participants sort stimuli in a specific number of groups. At the group-average level, free sorts were maximally correlated with the six-group derived constrained sorts. Notably, the number of groups created by participants in the free sorting condition was not significantly different than 6 (mean $=6.15, \mathrm{STD}=2.50$, $\mathrm{t}(39)=0.38, p=.76)$ : the group-average free sorts were thus maximally correlated with the derived constrained sorts based on the same number of groups. Still at the group-average level, the proportion of variance shared by free and six-group constrained sorts approaches the proportion of variance shared by the free sorts from two separate groups of 20 participants each $\left(\mathrm{R}^{2}=.67\right.$ and .74 , respectively, see Figure 2$)$ : the amount of variance shared by free and six-group sorts is thus comparable to what is expected for 
separate individuals that carry out the same free sorting task. Finally, we considered the $\mathrm{R}^{2}$ between group-average constrained sorts and the individual-level free sorts for the same sound set. We thus computed the absolute difference between the number of groups in each of the free sorts and in the various constrained sorts (e.g., absolute difference $=0$ for free and constrained sorts based on the same number of groups), and averaged $\mathrm{R}^{2}$ values between free and constrained sorts within each level of the absolute difference in the number of groups. Based on this analysis, the free sorts appeared to be maximally correlated with the derived constrained sorts based on; approximately; the same number of groups (Figure 4). Overall, these analyses indicate that the derived constrained sorts are an acceptable model of real constrained sorts.

\section{[Figure 4 here]}

We observed a very high proportion of variance shared between the group-average hierarchical sorts and the seven-group constrained sorts $\left(\mathrm{R}^{2}=.95\right)$. One potential conclusion to be drawn from this result is that part of the initial merging steps of a complete hierarchical sort are not necessary because they have a weak influence on the between-stimulus dissimilarities. To address this issue, we measured the redundancy $\left(\mathrm{R}^{2}\right.$ averaged across sound sets) between group-average complete hierarchical sorts and various truncated hierarchical sorts each derived by discarding a different number of the initial merging steps (Figure 5). The derived truncated sorts share a very high proportion of variance with the complete hierarchical sort even when the number of starting groups is less than half of the experimental stimuli $\left(\mathrm{R}^{2}>.95\right)$. For this reason, truncated hierarchical sorting variants with a relatively low number of starting groups are an advisable alternative to complete hierarchical sorting because their increased efficiency does not appear to come at a considerable loss in the amount of dissimilarity information.

[Figure 5 here] 
Data modeling. We investigated the effect of data-collection method on the change in fit of various distance models (see Table 3; and Appendix). An initial group of analyses, carried out with group-average data, considered a large number of distance models varying in the number of free parameters. The assessment of model fit in this initial step allowed us to address some standing issues concerning the number of free parameters in set-theoretic models. The quantification of models redundancy, i.e., the extent to they yield equivalent distances, allowed us to identify groups of largely diverse distance models. Based on this initial step, we selected a smaller set of distance models that had (approximately) the same number of free parameters and were characterized by a comparatively lower redundancy. The second group of analyses, carried out with group-average and individual data, assessed in detail the effects of method on the fit of the selected models. This analysis was complemented with a study of the effect of the distributional properties of the dissimilarities on model fit. The goal of this analysis was to explain divergences between results for group-average and individual data and to allow the experiment designer to better predict the effects of method on model fit.

[Table 3 here]

We fit various distance models to group-average data from the different methods, including the constrained sorts. We considered variants/derivations of seven basic distance models (see Table 3 for model class, interpretation in terms of common, distinctive, and unique features, and naming conventions): 1) the modified contrast model of Navarro and Lee (2004, MCM); 2) the additive clustering model of Shepard and Arabie (1979, ADCLUS); 3) the distinctive-features clustering model of Navarro and Lee (2004, DFCLUS); 4) the minimum variance root additive tree model (Sattath \& Tversky, 1977), estimated using the generalized triples algorithm of Corter (1998, GTREE); 5) the least-squares ultrametric tree (Hubert, Arabie, \& Meulman, 2006, L2ULTRA); 6) the 
centroid metric model (Barthélemy \& Guénoche, 1991, CENM); and 7) a nonmetric multidimensional scaling model (Takane, Young, \& De Leeuw, 1977, ALSCAL). We fit three variants for each of the MCM, ADCLUS and DFCLUS models by manipulating the number of nonuniversal features: 2,3 or 20 . Our manipulation of the number of features reflects the absence of a wide consensus on the number of free parameters for this class of models (see Appendix). From each of the MCM and additive-tree models, we derived both a common- and distinctive-feature metric. Two different centroid-distance metrics were fit either to the observed dissimilarities (CENM) or to their square (CENMSQ; see Equations 6 and 7 in Appendix). Finally, we fit the ALSCAL model with either two or three dimensions.

The ALSCAL model was fit using the secondary approach to the handling of tied ordinal data, which allows different model distances for input dissimilarities of the same modulus (Takane et al., 1977). The primary approach to ties, which attempts to assign the same model distance to tied input data, was not considered, because it is prone to annular and horseshoe biases (Goodhill et al., 1995). With the exception of CENM and CENMSQ, which have an exact least-squares solution, all models involve iterative criterion-minimization routines and are thus potentially prone to local minima problems (i.e., the fitting routines are not always guaranteed to converge on a globally optimal solution). We made an attempt at mitigating these problems by using a permutation approach for the input data. In particular, each of the models was fit 200 times to random permutations of the order of the stimuli within the dissimilarity matrices. The final solution minimized a criterion across the permutations: SSTRESS for ALSCAL and the squared error for the other models. In the following, we measure model fit as the $\mathrm{R}^{2}$ between input and model distances. When an MCM model included only common/distinctive features, the $\mathrm{R}^{2}$ for the distinctive/common component of the same model was set to zero. 
Across the 21 methods and 22 models, fit was higher for the semantic than for the synthetic set, $\mathrm{R}^{2}=.56$ and .52 , paired samples $t(461)=6.0, p<.001$, Cohen's $\mathrm{d}$ for paired samples t-test $=0.28$. This difference might be caused by a slightly higher reliability of the behavioral data for the semantic than for the synthetic set, where more reliable data are likely to be less influenced by measurement error, and thus to contain a large portion of variance that can be captured with a distance model. A good consistency was nonetheless observed between the effects of method on model fit for the two sound sets, $r(460)=.89, p<.001$. Further analyses averaged across sound sets. Figure 6 (left panel) shows the average model-specific fit across methods and the standard error of this measure. Note that the standard error of these fit quantities measures the strength of the effect of data-collection method on model fit. The data-collection method thus appears to affect most strongly the fit of the distinctive-feature models ALSCAL and DFCLUS, and that of the common- and distinctive-feature components of the MCM models. In general, and with the exception of CENM and CENMSQ, the effect of method on fit is weaker for models that, overall, are better fitting. As a rule of thumb, the data analyst should thus carefully consider the potential effects of data-collection method on model-based conclusions when models explain less than $70 \%$ of the variance of the group-average dissimilarities.

This initial analysis can also inform the debate on the number of free parameters in set-theoretic models. Across methods, all the 20-feature set-theoretic models reach an almost perfect fit. Since this result is potentially the product of overfitting (the model has so many free parameters that it also captures measurement noise), then these models likely have a very large number of parameters. Notably, according to Chaturvedi and Carroll (2006) each of these models have $N$ stimuli $\times K$ features $+1=401$ parameters whereas according to Carroll and Arabie (1983) and Shepard and Arabie (1979) they have $N+K+1=41$ or $K+1=21$ free parameters, respectively. As such, only the position 
by Chaturvedi and Carroll (2006) appears to account for the overfit of the 20-feature models. Another result potentially consistent with the position of Chaturvedi and Carroll (2006) is the fact that for all set-theoretic models an increase in the number of features from 2 to 3 (from 23 to 24 parameters according to Carroll \& Arabie, 1983, and from 41 to 61 parameters according to Chaturvedi \& Carroll, 2006) explains $10 \%$ of the variance in the input data. Note that for the ALSCAL model a similar improvement in explained variance is achieved by 19 additional parameters (compare the fit for the two- and three dimensional ALSCAL models), a figure similar to the number of additional parameters assumed by Chaturvedi and Carroll (2006) for ADCLUS. For these reasons, in the following we will adopt the position of Chaturvedi and Carroll (2006) as a working solution to the debate on the number of parameters in set-theoretic models.

\section{[Figure 6 here]}

We measured the redundancy of the distance estimates from different models. For each of 42 datasets (21 data-collection methods $\times 2$ sound sets), we defined a matrix of measures of between-model redundancy as the $\mathrm{R}^{2}$ between the distance estimates of each of the 22 distance models. We took the average of the redundancy matrices across sound sets and data-collection methods and fit a two-dimensional metric MDS model (ALSCAL) to a distance metric defined as $1-\mathrm{R}^{2}$ (see Figure 6 , right panel; ALSCAL $\mathrm{R}^{2}=.83$ ). Based on this MDS analysis, the distance models appear to form three separate clusters: 1) the unique-feature models CENM and CENMSQ; 2) the distinctive-feature models $\mathrm{ALSCAL}_{3}$, $\mathrm{ALSCAL}_{2}, \mathrm{DFCLUS}_{3}$ and $\mathrm{DFCLUS}_{2}$, and $\mathrm{MCM}_{\mathrm{D}}$; and 3) the common-features models

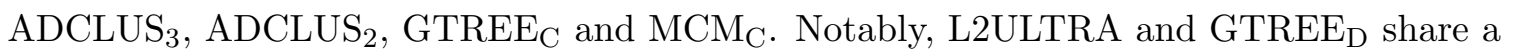
high portion of variance with $\mathrm{GTREE}_{\mathrm{C}}$. This result might be the product of the overall poor fit of the centroid metric, which produces an additive tree in which the objects are equidistant from the root (a defining property of ultrametric trees), and a complementarity 
of the common and distinctive metrics of the additive tree (see Appendix). Finally, the models $\mathrm{MCM}_{20}, \mathrm{ADCLUS}_{20}$ and $\mathrm{DFCLUS}_{20}$, which are likely to overfit the data, lie in a region intermediate between the common- and distinctive-features clusters, a region also occupied in part by the hybrid common/distinctive feature models $\mathrm{MCM}_{2}$ and $\mathrm{MCM}_{3}$.

We analyzed in detail the effect of data-collection method on the fit of a subset of the distance models (see Figure 7). Based on the results of the initial analyses, we selected the following models (number of parameters): $\operatorname{ADCLUS}_{2}(38), \operatorname{GTREE}_{\mathrm{D}}(37), \mathrm{DFCLUS}_{2}$ (41), ALSCAL $_{2}$ (41) and CENM (20). These models appear to span the entire MDS space of distance models (see Figure 6), and, with the exception of the CENM model, all have approximately the same number of parameters. The distance models were fit to both group-average and individual data. Prior to MDS fitting, binary individual dissimilarities (free and constrained sorts) were $\delta$-transformed (Rosenberg \& Kim, 1975):

$\delta_{i j}=\left\{\left(\sum_{k} d_{i k}-d_{j k}\right)^{2}\right\}^{1 / 2}$. The $\delta$ transform decreases the strength of horseshoe/annular biases in nonmetric MDS (Goodhill et al., 1995), and does not alter the accuracy of MDS models of noisy data such as the behavioral dissimilarities from this study (Dragsgow \& Jones, 1979). For consistency, binary dissimilarities were $\delta$ transformed prior to fitting any model. All models were fit by using the above-described permutation approach.

\section{[Figure 7 here]}

Focusing on group-average data (Figure 7, left panel), the fit of all methods appears relatively constant across the dissimilarity ratings and hierarchical and free sorting methods. An exception to this is the fit for CENM, which is much higher for dissimilarity ratings than for both hierarchical and free sorting. The fit of the common- and unique-feature models is also relatively constant across constrained sorts and shows overall higher levels of fit for $\mathrm{GTREE}_{\mathrm{D}}$ than for $\mathrm{ADCLUS}_{2}$ than for CENM. Consistently with the initial analyses, the fit of the distinctive-feature models $\mathrm{DFCLUS}_{2}$ and $\mathrm{ALSCAL}_{2}$ 
appears to change strongly across constrained sorts. It increases progressively as participants merge larger and larger groups of stimuli and passes through the point where common- and distinctive-features models explain the same amount of variance in the data (5- to 8-group constrained sorts). Thereafter, these models yield better fits than common-feature models. From a psychological standpoint, these results suggest that common features are equally important throughout all the levels of a cognitive taxonomy, whereas distinctive features dominate the superordinate levels. Furthermore, the constrained sorting data for which the common-features model ADCLUS 2 and the distinctive-features models $\mathrm{DFCLUS}_{2}$ and $\mathrm{ALSCAL}_{2}$ explain equal proportions of variance are also those that share the largest proportion of variance with dissimilarity ratings and hierarchical and free sorting data (cf. Figure 3). We have no explanation for this result. Different trends emerge from the analysis of individual data (Figure 7, right panel). When compared with group-average results, larger variations in fit emerge across dissimilarity ratings and hierarchical and free sorts. Across models, the fit for free sorts is better than for hierarchical sorts and dissimilarity ratings, with the exception of the unsurprising perfect fit of $\mathrm{GTREE}_{\mathrm{D}}$ for hierarchical sorts. Three results emerge from the analysis of individual constrained sorts. Firstly, the unique-features model (CENM) explains a larger proportion of variance for individual than for group-average data, with fits that progressively decrease as participants merge larger and larger groups of stimuli. Secondly, the fit of both the common- and distinctive-features models varies across the constrained sorts. Finally, the fit of all models follows a U-shaped function of the number of groups in the constrained sorting data.

One potential explanation for the difference of results across group-average and individual data focuses on the violation of the triangle inequality, a metric axiom according to which the distance between objects $\mathrm{A}$ and $\mathrm{B}$ is always equal to or less than the sum of the distances of $\mathrm{A}$ and $\mathrm{B}$ from a third object. This metric axiom is implicit in the MDS 
and DFCLUS models and in all graph-theoretic models, but not in ADCLUS (Tversky, 1977; Navarro \& Lee, 2004; Sattath \& Tversky, 1977). In particular, Ashby, Maddox, and Lee (1994) showed that the averaging process decreases the number of violations of the triangle inequality, and improves the fit of MDS models compared to what is observed for individual data. Consistently with this interpretation, group-average dissimilarity ratings were characterized by fewer violations than were individual data (average number of violations $=0.02$ and $=0.32$, respectively). Notably, this explanation does not account for the results for sorting data because, by definition, they satisfy the triangle inequality at both the group-average and individual levels. Another explanation for the different results for group-average and individual data focuses on the distributional properties of the input dissimilarities and on the sensitivity of the distance models to such variations (Pruzansky et al., 1982; Ghose, 1998). We thus assessed the extent to which models fit was influenced by the skewness and elongation (proportion of elongated triangles in the distance matrix) of the input data. For each of the distance models, we computed a multiple rank-regression model (Iman \& Conover, 1979), with model fit as dependent variable and skewness and elongation as predictors (Table 2). We considered group-average and individual data together. To consider the same number of group-average and individual datapoints, model fit, skewness and elongation were averaged across individuals. Within the rank-regression model, the strength of the effect of the predictors was measured by their partial $\mathrm{R}^{2}\left(\mathrm{R}_{\mathrm{p}}^{2}\right.$ ) within the multivariate model (Mulaik, 2005), as computed based on the observed values of model fit rather than on the ranked values.

[Table 2 here]

Overall, data skewness and elongation explained the variations in the fit of distinctive-features models better than those of common-features models, with intermediate levels of explained variance for the unique-features model. Consistently with 


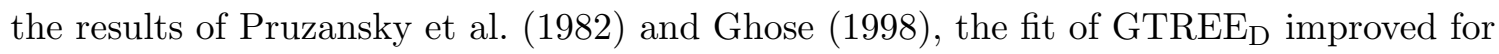
lower skewness values, whereas that of all the other models, $\mathrm{ALSCAL}_{2}$ included, improved for higher skewness values. Although CENM fits datasets with low elongation better, all of the other models yielded better fits for high elongation values. Notably, previous studies reported better additive tree and MDS fits for high and low values of elongation, respectively (Pruzansky et al., 1982; Ghose, 1998). Our results for the ALSCAL 2 model are thus contrary to this trend. We have no explanation for this difference. Finally, as revealed by the $\mathrm{R}_{\mathrm{p}}^{2}$ values, the fit of distinctive- and common-features models is better explained by variations in skewness and elongation, respectively. To a first approximation, the fit of the unique-features model is instead equally well explained by variations in both factors. Among the various results of this analysis, the stronger ones concern the effect of skewness on the fit of distinctive-features models $\left(\mathrm{R}^{2} \geq .80\right)$. Accordingly, the experiment designer should be particularly aware of the extent to which the distributional properties of the data yielded by the chosen method influences the ability to fit distinctive-features models.

Accuracy. We measured the accuracy of the group-average and individual data from the different data-collection methods. Accuracy was defined as the extent to which the behavioral data allow recovery of a distance metric based on the stimulus features. We focused on the data collected with the synthetic sounds, because their features were known in advance: attack time, spectral centroid and energy ratio between even and odd harmonics. Accuracy was defined as the $\mathrm{R}^{2}$ between behavioral dissimilarities and a Euclidean measure of the between-stimuli distance based on the stimulus features. For the sake of coherence with the above-presented analyses, individual data were $\delta$-transformed. Figure 8 shows the $\mathrm{R}^{2}$ values between feature distances and group-average and individual data. Because of the fact that accuracy was measured with reference to a Euclidean distance based on the features, between-methods differences in accuracy might be influenced by the 
ability to fit a Euclidean structure to the various behavioral datasets. However, alternative accuracy measures based on alternative metrics of the feature-based distance (e.g., additive tree) produced the same results; and are not shown here for the sake of brevity.

\section{[Figure 8 here]}

Several points emerge from this analysis. Firstly, and not surprisingly, less noisy group-average data are more accurate than individual data. Secondly, and consistently with previous studies, dissimilarity rating is by far the most accurate method (Bricker \& Pruzansky, 1970; Subkoviak \& Roecks, 1976). Thirdly, free sorting and hierarchical sorting are equally accurate at the group-average level, whereas hierarchical sorts are more accurate at the individual level. The first of these results is in contrast with the superior accuracy of group-average hierarchical sorts compared to free sorts observed by Rao and Katz (1971). Several methodological differences might explain this inconsistency. For example, Rao and Katz (1971) assumed a Euclidean mental space and measured accuracy in MDS models fit to the dissimilarities. Our datasets were better fit with graph-theoretic structures, and the analysis of accuracy focused on the raw data. Among the various factors, a particular aspect of the free sorts simulated by Rao and Katz (1971) appears to provide a straightforward explanation for the divergence. The maximum number of free-sorting groups is proportionally lower than what was observed with the participants in our experiment (8 groups $/ 40$ stimuli $=0.2$ for Rao \& Katz, $1971 ; 6.15$ groups $/ 20$ stimuli $=0.31$ in this study). As such, the free sorting data from their study are likely more comparable to the four-groups constrained sorts than to the free sorts from the current study (4 groups/20 stimuli $=0.2$ ). Based on these considerations, our results are consistent with those of Rao and Katz (1971) at both the group-average and individual levels: in both cases, the four-group constrained sorts are less accurate than the hierarchical sorts. The superior accuracy of individual sorts also 
provides at least partial support for the hypothesis that hierarchical sorting produces data that are more appropriate than does free sorting for individual-differences scaling (Lawless et al., 1995). Indeed, more accurate individual data are more likely to yield interpretable solutions for individual-differences models.

\section{Conclusions}

We compared dissimilarity ratings and sorting methods relative to a variety of factors of potential relevance to the experiment design process: efficiency, reliability, between-method redundancy, data modeling and accuracy. Table 4 ranks the various methods relative to most of these criteria.

\section{[Table 4 here]}

Consistently with previous studies, dissimilarity ratings scored as a highly inefficient method for large stimulus sets, whereas free sorting was drastically more efficient. When compared to dissimilarity ratings, the gain in efficiency associated with hierarchical sorting appeared to be minimal if participants were asked to create the entire hierarchy. Interestingly, modeling results showed that the truncated hierarchical sorting methods are at least as efficient as free sorting. The analysis of reliability revealed an efficiency-reliability tradeoff: less efficient methods that required participants to inspect each stimulus a larger number of times produced more reliable data, more likely to be replicated with different groups of participants. Dissimilarity ratings and free sorting were thus the most and least reliable methods, respectively, with an intermediate reliability for hierarchical sorting. Similar results emerged from the analysis of data accuracy: dissimilarity ratings reflected the stimulus features more closely than any of the sorting methods at both the group-average and individual levels. Partially mitigating the plausible hypothesis of an efficiency-accuracy tradeoff, whereas hierarchical sorting was more accurate than free 
sorting at the individual level, both methods appeared equally accurate at the group-average level.

The analysis of cross-method redundancy revealed that group-average dissimilarity ratings and hierarchical and free sorting dissimilarities share a considerable amount of variance, approximately $60 \%$. These results might in principle support the choice of more efficient sorting methods over dissimilarity ratings. This choice should nonetheless considering the lower accuracy and reliability of sorting methods. Because of these latter properties, sorting methods should be adopted with extreme parsimony and only when strictly necessary (e.g., strong adaptation effects; measurement of context effects vulnerable to long dissimilarity-estimation sessions). The choice of sorting methods should be cautious even when dealing with large sets of stimuli. In such cases, and depending on the available resources, the experimenter might thus still opt for dissimilarity ratings and distribute the judgment of the various pairs of stimuli across different-day experimental sessions, and collect multiple ratings of each of the pairs from each of the participants. In the absence of the necessary conditions, truncated hierarchical sorting should be considered as the best alternative to dissimilarity ratings. Redundancy analyses showed that truncated hierarchical sorts contain a very large amount of information about the complete-hierarchical sorts even when the starting number of groups is less than one third of the number of experimental stimuli. For this reason, truncated hierarchical sorting is highly likely to keep the higher individual-level accuracy and reliability of complete hierarchical sorts while at the same time reaching similar efficiency levels as free sorting.

Overall, the analysis of data-modeling biases revealed that the fit of distinctive-features models such as MDS is particularly sensitive to a change in data-collection methods. This effect appears to be strongly dependent on the skewness of the dissimilarities. In particular, and consistently with previous studies, distinctive-feature models better fit data with a moderately negative to positive skewness. Given the 
relatively strong dependence of model fit on the data-collection method, it is recommended to carry out analyses based on the raw unmodeled dissimilarities as frequently as possible. In the case of strong interest for distance models, the experimenter is advised to evaluate the robustness of the main conclusions against variations in the data-collection method, and against variations in the distance model itself (e.g., test whether both MDS and additive tree models of the same data suggest the perceptual relevance of the same stimulus features). 


\section{References}

Arabie, P., Carroll, J. D., \& DeSarbo, W. (1987). Three-way scaling and clustering. Newbury Park, CA: Sage Publications.

Ashby, F., Maddox, W., \& Lee, W. (1994). On the dangers of averaging across subjects when using multidimensional scaling or the similarity-choice model. Psychological Science, 5(3), 144-151.

Barthélemy, J. P., \& Guénoche, A. (1991). Trees and proximity representations.

Chichester, UK: John Wiley and Sons Ltd.

Bertino, M., \& Lawless, H. T. (1993). Understanding mouthfeel attributes: A multidimensional scaling approach. Journal of Sensory Studies, 8(2), 101-114.

Bijmolt, T. H. A., \& Wedel, M. (1995). The effects of alternative methods of collecting similarity data for multidimensional scaling. International Journal of Research in Marketing, 12(4), 363-371.

Bimler, D. L., \& Kirkland, J. (1997). Multidimensional scaling of hierarchical sorting data applied to facial expressions. Scandinavian Journal of Psychology, 38(4), 349-357.

Bimler, D. L., Kirkland, J., \& Chen, S.-J. (1998). A cross-cultural study of facial expressions of emotion using multidimensional scaling (Annual Report No. 21). Research and Clinical Center for Child Development, Hokkaido University.

Bonebright, T. L. (1996). An investigation of data collection methods for auditory stimuli: Paired comparisons versus a computer sorting task. Behavior Research Methods, Instruments, and Computers, 28(2), 275-278.

Borg, I., \& Groenen, P. (1997). Modern multidimensional scaling. New York, NY: Springer-Verlag.

Boster, J. (1986). Can individuals recapitulate the evolutionary development of color lexicons? Ethnology, 25(1), 61-74.

Bricker, P. D., \& Pruzansky, S. (1970). Comparison of sorting and pairwise similarity 
judgment techniques for scaling auditory stimuli. Journal of The Acoustical Society Of America, 47(1A), 96-96.

Caclin, A., McAdams, S., Smith, B. K., \& Winsberg, S. (2005). Acoustic correlates of timbre space dimensions: A confirmatory study using synthetic tones. Journal of The Acoustical Society Of America, 118(1), 471-482.

Carroll, J. D. (1976). Spatial, non-spatial and hybrid models for scaling. Psychometrika, 41(4), 439-463.

Carroll, J. D., \& Arabie, P. (1983). INDCLUS: An individual differences generalization of the ADCLUS model and the MAPCLUS algorithm. Psychometrika, 48(2), 157-169.

Carroll, J. D., \& Pruzansky, S. (1980). Discrete and hybrid scaling models. In E. D. Lantermann \& H. Feger (Eds.), Similarity and choice, papers in honour of Clyde Coombs (p. 108-139). Bern, Switzerland: Hans Huber Publishers.

Cartier, R., Rytz, A., Lecomte, A., Poblete, F., Krystlik, J., Belin, E., et al. (2006). Sorting procedure as an alternative to quantitative descriptive analysis to obtain a product sensory map. Food Quality and Preference, 17(7-8), 562-571.

Chaturvedi, A., \& Carroll, J. D. (2006). CLUSCALE ("CLUstering and multidimensional SCAL[E]ing"): A three-way hybrid model incorporating overlapping clustering and multidimensional scaling structure. Journal of Classification, 23(2), 269-299.

Corter, J. E. (1996). Tree models of similarity and association. Thousand Oaks, CA: Sage Publications.

Corter, J. E. (1998). An efficient metric combinatorial algorithm for fitting additive trees. Multivariate Behavioral Research, 33(2), 249-71.

Coxon, A. P. M. (1999). Sorting data: Collection and analysis. Thousand Oaks, CA: Sage Publications.

Dragsgow, F., \& Jones, L. E. (1979). Multidimensional scaling of derived dissimilarities. Multivariate Behavioral Research, 14(2), 227-244. 
Efron, B., \& Tibishirani, R. J. (1993). An introduction to the bootstrap. New York, NY: Chapman and Hall.

Gati, I., \& Tversky, A. (1982). Representations of qualitative and quantitative dimensions. Journal of Experimental Psychology: Human Perception and Performance, 8(2), 325-40.

Ghose, S. (1998). Distance representations of consumer perceptions: Evaluating appropriateness by using diagnostics. Journal of Marketing Research, 35(2), $137-153$.

Giordano, B. L., McDonnell, J., \& McAdams, S. (2010). Hearing living symbols and nonliving icons: Category-specificities in the cognitive processing of environmental sounds. Brain and Cognition, 73, 7-19.

Goldstone, R. L. (1994). The role of similarity in categorization: providing a framework. Cognition, 52, 125-157.

Goldstone, R. L. (1999). Similarity. In R. A. Wilson \& F. C. Keil (Eds.), MIT encyclopedia of the cognitive sciences (pp. 763-764). Cambridge, MA: MIT Press.

Goodhill, G. J., Simmen, M. W., \& Willshaw, D. J. (1995). An evaluation of the use of multidimensional scaling for understanding brain connectivity. Philosophical Transactions of the Royal Society B: Biological Sciences, 348(1325), 265-280.

Graef, J., \& Spence, I. (1979). Using distance information in the design of large multidimensional scaling experiments. Psychological Bulletin, 86(1), 60-66.

Griffiths, T. L., \& Kalish, M. L. (2002). A multidimensional scaling approach to mental multiplication. Memory and Cognition, 30(1), 97-106.

Harbke, C. R. (2003). Evaluation of data collection techniques for multidimensional scaling with large stimulus sets. Unpublished master's thesis, Washington State University, Department of Psychology.

Harloff, J., \& Coxon, A. P. M. (2005). How to sort: A short guide on sorting 
investigations. Available from http://www.methodofsorting.com

Henry, W. A., \& Stumpf, R. V. (1975). Time and accuracy measures for alternative multidimensional scaling data collection methods. Journal of Marketing Research, 12(2), 165-170.

Holman, E. W. (1972). The relation between hierarchical and euclidean models for psychological distances. Psychometrika, 37(4), 417-423.

Hubert, L. J., Arabie, P., \& Meulman, J. (1997). Linear and circular unidimensional scaling for symmetric proximity matrices. British Journal of Mathematical and Statistical Psychology, 50(2), 253-284.

Hubert, L. J., Arabie, P., \& Meulman, J. (2006). The structural representation of proximity matrices with MATLAB. SIAM, Philadelphia, PA, ASA, Alexandria, VA.

Iman, R., \& Conover, W. (1979). The use of the rank transform in regression. Technometrics, 21(4), 499-509.

ISO. (2004). Acoustics - Reference zero for the calibration of audiometric equipment Part 8: Reference equivalent threshold sound pressure levels for pure tones and circumaural earphones (ISO 389-8) (Tech. Rep.). International Organization for Standardization, Geneva.

Johnson, M. D., Lehmann, D. R., \& Horne, D. R. (1990). The effects of fatigue on judgments of interproduct similarity. International Journal of Research in Marketing, 7(1), 35-43.

Johnson, S. C. (1967). Hierarchical clustering schemes. Psychometrika, 32(3), 241-254.

Kendall, D. G. (1975). The recovery of structure from fragmentary information. Philosophical Transactions for the Royal Society of London. Series A, Mathematical and Physical Sciences, 279(1291), 547-582.

Kroonenberg, P. M. (2008). Applied multiway data analysis. Hobokn, NJ: John Wiley and Sons, Inc. 
Lawless, H. T., Sheng, N., \& Knoops, S. S. C. P. (1995). Multidimensional scaling of sorting data applied to cheese perception. Food Quality and Preference, 6(2), 91-98.

Malhotra, N. K. (1990). Comment on: "The effects of fatigue on judgments of interproduct similarity", by M. D. Johnson, D. R. Lehmann and D. R. Horne. International Journal of Research in Marketing, 7, 45-51.

Martin, F. N., \& Champlin, C. A. (2000). Reconsidering the limits of normal hearing. Journal of The American Academy Of Audiology (Hamilton, Ont., 11(2), 64-66.

Miller, G. A. (1969). A psychological method to investigate verbal concepts. Journal of Mathematical Psychology, 6(2), 169-191.

Miller George, A. (1956). The magical number seven, plus or minus two: some limits on our capacity for processing information. The Psychological Review, 63, 81-97.

Mulaik, S. (2005). Variable selection. In B. S. Everitt \& D. C. Howell (Eds.), Encyclopedia of statistics in behavioral science. Chichester, UK: John Wiley and Sons, Ltd.

Navarro, D. J., \& Lee, M. D. (2004). Common and distinctive features in stimulus similarity: A modified version of the contrast model. Psychonomic Bulletin and Review, 11(6), 961-974.

Pruzansky, S., Tversky, A., \& Carroll, J. D. (1982). Spatial versus tree representations of proximity data. Psychometrika, 47(1), 3-24.

Rao, V. R., \& Katz, R. (1971). Alternative multidimensional scaling methods for large stimulus sets. Journal of Marketing Research, 8(4), 488-494.

Rosenberg, S., \& Kim, M. P. (1975). The method of sorting as a data-gathering procedure in multivariate research. Multivariate Behavioral Research, 10(4), 489-502.

Sattath, S., \& Tversky, A. (1977). Additive similarity trees. Psychometrika, 42(3), $319-345$

Shepard, R. N. (1962a). The analysis of proximities: Multidimensional scaling with an unknown distance function. I. Psychometrika, 27(2), 125-140. 
Shepard, R. N. (1962b). The analysis of proximities: Multidimensional scaling with an unknown distance function. II. Psychometrika, 27(3), 219-246.

Shepard, R. N. (1987). Toward a universal law of generalization for psychological science. Science, 237(4820), 1317-1323.

Shepard, R. N., \& Arabie, P. (1979). Additive clustering: Representation of similarities as combinations of discrete overlapping properties. Psychological Review, 86(2), $87-123$.

Simmen, M. W. (1996). Multidimensional scaling of binary dissimilarities: Direct and derived approaches. Multivariate Behavioral Research, 31(1), 47-67.

Spence, I., \& Domoney, D. W. (1974). Single subject incomplete designs for nonmetric multidimensional scaling. Psychometrika, 39(4), 469-490.

Subkoviak, M. J., \& Roecks, A. L. (1976). A closer look at the accuracy of alternative data-collection methods for multidimensional scaling. Journal of Educational Measurement, 13(4), 309-317.

Takane, Y., Young, F. W., \& De Leeuw, J. (1977). Nonmetric individual differences multidimensional scaling: An alternating least squares method with optimal scaling features. Psychometrika, 42(1), 7-67.

Torgerson, W. S. (1952). Multidimensional scaling: I. Theory and method. Psychometrika, 17(4), 401-419.

Tsogo, L., Masson, M. H., \& Bardot, A. (2000). Multidimensional scaling methods for many-object sets: A review. Multivariate Behavioral Research, 35(3), 307-319.

Tversky, A. (1977). Features of similarity. Psychological Review, 84(4), 327-352.

Tversky, A., \& Gati, I. (1978). Studies of similarity. In E. Rosch \& B. B. Lloyd (Eds.), Cognition and categorization (pp. 79-98). Mahwah, NJ: Erlbaum.

Ward, L. M. (1977). Multidimensional scaling of the molar physical environment. Multivariate Behavioral Research, 12(1), 23-42. 
COMPARISON OF METHODS FOR COLLECTING AND MODELING DISSIMILARITIES 37

Winsberg, S., \& Carroll, J. D. (1989). A quasi-nonmetric method for multidimensional scaling via an extended Euclidean model. Psychometrika, 54, 217-229. 


\section{Appendix: Distance models}

Distance models can be classified into three categories: spatial models, set-theoretic models and graph-theoretic models (Corter, 1996). All of the considered models assume that the distance between objects is a function of their features, which can be of three types: common features, which increase the similarity of the objects that share them; distinctive features, which increase the dissimilarity of the objects that do not share them (Tversky, 1977); unique features, or specificities (Winsberg \& Carroll, 1989), a special type of distinctive feature that characterizes only one of the objects in the set.

The distance models presented here are appropriate for the analysis of two-way data. Three-way extensions of part of the models presented here are described in Arabie, Carroll, and DeSarbo (1987), Kroonenberg (2008), and Carroll and Arabie (1983).

Spatial models. Spatial models represent the dissimilarity $d_{i j}$ between objects $i$ and $j$, with $i, j=1, \ldots, N$, as their distance $d_{i j}^{\prime}$ in a space. In the multidimensional scaling (MDS) model

$$
d_{i j}^{\prime}=\left[\sum_{d=1}^{D}\left(x_{i d}-x_{j d}\right)^{r}\right]^{1 / r}
$$

where $D$ is the number of dimensions, $x_{i d}$ is the coordinate of object $i$ along the $d$ th dimension, and $r$ is the power of the Minkowski metric. The classical MDS model is

metric and the space is Euclidean, i.e., $d_{i j}^{\prime}$ is a linear function of $d_{i j}$ and $r=2$ (Torgerson, 1952). In the nonmetric MDS model, $d_{i j}^{\prime}$ is more generally a monotonic function of $d_{i j}$ (Shepard, 1962a, 1962b). Taking into account the translational invariance of MDS models, i.e., the fact that the configuration can be translated arbitrarily without affecting the distances, the number of free parameters equals $D(N-1)$. MDS can be considered a distinctive-features model, because features common to all objects (e.g., shape for triangles of different colors) do not affect their distance (Gati \& Tversky, 1982). 
Set-theoretic models. In this class of models, objects are conceived as collections of features. One of the most general formulations is the contrast model (Tversky, 1977). In a recent variant of the contrast model, the modified contrast model (MCM, Navarro \& Lee, 2004), the between-objects proximity $s_{i j}^{\prime}$ equals

$$
\begin{aligned}
s_{i j}^{\prime} & =c+s c_{i j}^{\prime}+s d_{i j}^{\prime} \\
s c_{i j}^{\prime} & =\sum_{k \in C F} w_{k} f_{i k} f_{j k} \\
s d_{i j}^{\prime} & =-\frac{1}{2} \sum_{k \in D F} w_{k} f_{i k}\left(1-f_{j k}\right)-\frac{1}{2} \sum_{k \in D F} w_{k} f_{j k}\left(1-f_{i k}\right)
\end{aligned}
$$

where $c$ is a constant term modeling the saliency of a universal feature common to all stimuli (Shepard \& Arabie, 1979), $s c_{i j}^{\prime}$ and $s d_{i j}^{\prime}$ are the common- and distinctive-feature proximity metrics, $f=(1, \ldots, K)$ is a feature that can be either common $(C F)$ or distinctive $(D F)$ and $w_{k}$ is the weight of the $k$ th feature. The MCM is a hybrid common/distinctive features model. It reduces to the common-features additive-clustering model (ADCLUS, Shepard \& Arabie, 1979) and to the distinctive-features model (DFCLUS, Navarro \& Lee, 2004) if $D F=\emptyset$ or $C F=\emptyset$, respectively. Current opinions diverge on the number of free parameters in a set-theoretic model. For ADCLUS, this number equals $K+1$ according to Shepard \& Arabie, 1979; see also Navarro \& Lee, 2004, p. 966), $N+K+1$ according to Carroll and Arabie (1983) and $N K+1$ according to Chaturvedi and Carroll (2006). The disagreement likely extends to DFCLUS and MCM.

Graph-theoretic models. Graph-theoretic structures model distances as the minimum-length path between nodes, each representing an object. A path is a sequence of arcs (e.g., Figure 3). A particularly important graph-theoretic structure is the tree, i.e., a graph that is connected (each pair of nodes is connected by a path) and does not have cycles (each path passes through one node only once; Corter, 1996).

In the additive tree (Carroll, 1976; Sattath \& Tversky, 1977), distances satisfy the 
additive inequality:

$$
d_{i j}+d_{k l} \leq \max \left(d_{i k}+d_{j l}, d_{i l}+d_{j k}\right)
$$

implying that for each quadruple of objects, the two largest of the three sums in Equation 4 are equal (Barthélemy \& Guénoche, 1991, p. 50). In a rooted additive tree, all the paths descend from a common point (e.g., Figure 3). In principle, an additive tree can be rooted in infinitely many different ways, i.e., the root can be located on any point in the tree. Commonly, one uses the minimum-variance root, which minimizes the variance of its distances from all the objects (Sattath \& Tversky, 1977). Under this condition, the root can be thus taken as a representation of the stereotypical stimulus for the set. An additive-tree model requires the estimation of $2 N-3$ parameters (Sattath \& Tversky, 1977). Additive trees can yield both a distinctive-features dissimilarity and a common-features similarity metric. In the first widely known case, the minimum-path distance between two objects models the weight of unshared features (Tversky, 1977). In the second, frequently ignored, case, the length of an arc models the weight of the features common to the object that descend from the same arc (Tversky, 1977, p. 347). Note that whereas the distinctive-feature dissimilarity is independent of the location of the root, the common-features similarity depends on the location of the root.

Ultrametric trees, among which the hierarchical clustering model (S. C. Johnson, 1967), are a special case of the additive tree. In ultrametric trees, objects are equidistant from the root, and distances satisfy the ultrametric inequality:

$$
d_{i j} \leq \max \left(d_{i k}, d_{j k}\right)
$$

which implies that each possible triad of objects defines an isosceles triangle. An ultrametric tree is specified by $N-1$ parameters. As with additive trees, ultrametric trees can yield both a common-features proximity, the length of an arc from which two objects descend (Corter, 1996, p. 14), and a distinctive-features distance, the minimum-length 
path between objects. Differently from additive trees, however, the sum of these two metrics is the same for each pair of objects. As such, in an ultrametric tree it is not possible to distinguish between common- and distinctive-features metrics.

An additive tree with a single internal node is called a singular tree, (Sattath \& Tversky, 1977) or star tree or centroid distance (Barthélemy \& Guénoche, 1991, p. 88). With the centroid distance, the additive inequality reduces to the equality of all of the three sums in Equation 4 (Corter, 1996, p. 19), and distances are modeled as

$$
d_{i j}^{\prime}=x_{i}+x_{j}
$$

The least-squares estimation of the $x$ terms is given by:

$$
x_{i}=\frac{\sum_{j=1, j \neq i}^{N} d_{i j}+k}{N-2}-\frac{\sum_{i=1}^{N} \sum_{j=1, j \neq i}^{N} d_{i j}+k}{2(N-1)(N-2)}
$$

where $k$ is a constant term appropriately chosen to ensure positivity of $x_{i}$, with $i=1, \ldots, N$ (Hubert, Arabie, \& Meulman, 1997). A centroid distance estimates $N$ parameters. A formulation of the centroid distance similar to that in Equation 6 is used in EXSCAL, a hybrid of a tree and an MDS model (Winsberg \& Carroll, 1989). When the spatial component is null, EXSCAL reduces to:

$$
d_{i j}^{\prime}=\left[x_{i}+x_{j}\right]^{1 / 2}
$$

where the $x$ terms, termed specificities, weight the attributes specific to each of the stimuli. Independent of the particular formulation, the centroid distance can be interpreted as a unique-feature model. Overall, these models can be interpreted as a measure of the weight of features unique to each of the objects.

Note finally that an additive tree can always be decomposed in infinitely many ways as the sum of an ultrametric tree and a centroid metric (Barthélemy \& Guénoche, 1991, p. 114). The ambiguity of the decomposition reflects the fact that an additive tree can be 
rooted in infinitely many ways. As such, an additive tree can also be interpreted as the sum of a unique-features dissimilarity with an ultrametric tree, which might be equally well interpreted as a model of distinctive-features dissimilarity or of common-features similarity. 
Table 1

Temporal factors for the data-collection methods and sound sets averaged across participants (SE in parentheses).

\begin{tabular}{lcccccc}
\hline & \multicolumn{3}{c}{ Semantic set } & \multicolumn{3}{c}{ Synthetic set } \\
\cline { 2 - 7 } Method & DR & HS & FS & DR & HS & FS \\
\hline Experiment duration (min33.09(1.77) & $25.70(1.75)$ & $14.66(0.61)$ & $21.18(1.65)$ & $17.39(1.33)$ & $17.18(1.91)$ \\
Playback time (min) & $18.76(1.26)$ & $14.88(1.22)$ & $4.77(0.28)$ & $7.21(0.66)$ & $5.65(0.52)$ & $3.94(0.71)$ \\
Nonplayback time (min) & $14.33(0.91)$ & $10.82(0.61)$ & $9.90(0.53)$ & $13.97(1.11)$ & $11.74(0.87)$ & $13.24(1.36)$ \\
Number of playbacks & $23.05(1.55)$ & $18.40(1.51)$ & $5.96(0.36)$ & $33.96(3.11)$ & $27.11(2.51)$ & $19.28(3.41)$ \\
\hline
\end{tabular}


Table 2

Multiple rank regression analysis of the effects of data skewness and elongation on the fit of distance models.

\begin{tabular}{lcccccccc}
\hline & \multicolumn{3}{c}{ Skewness } & \multicolumn{5}{c}{ Elongation } \\
\cline { 2 - 7 } & $B$ & $S E B$ & $\mathrm{R}_{\mathrm{p}}^{2}$ & $B$ & $S E B$ & $\mathrm{R}_{\mathrm{p}}^{2}$ & $\mathrm{R}^{2}$ \\
\hline ADCLUS $_{2}$ & 0.19 & 0.10 & .05 & $0.32^{* *}$ & 0.10 & .08 & .10 \\
DFCLUS $_{2}$ & $0.84^{* *}$ & 0.05 & .78 & $0.22^{* *}$ & 0.05 & .17 & .80 \\
$\mathrm{CENM}$ & $0.53^{* *}$ & 0.08 & .70 & $-0.50^{* *}$ & 0.08 & .60 & .67 \\
GTREE $_{\mathrm{D}}$ & $-0.24^{*}$ & 0.10 & .12 & $0.46^{* *}$ & 0.10 & .21 & .24 \\
$\mathrm{ALSCAL}_{2}$ & $0.81^{* *}$ & 0.05 & .82 & $0.33^{* *}$ & 0.05 & .17 & .84 \\
\hline
\end{tabular}

Note. $\mathrm{B}=$ rank regression coefficient; $\mathrm{R}_{\mathrm{p}}^{2}=$ partial $\mathrm{R}^{2} ;{ }^{*} p<.05 ;{ }^{* *} p<.01 ; d f=81$. 
Distance models considered in this study.

\begin{tabular}{|c|c|c|c|}
\hline Acronym & Model & Model family & Feature interpretation \\
\hline$\S A_{L S C A L}$ & $\begin{array}{l}\text { Multidimensional scaling (alternating least-squares } \\
\text { algorithm); X = number of dimensions }\end{array}$ & Spatial & Distinctive \\
\hline$\dagger \mathrm{MCM}_{\mathrm{X}}$ & Modified contrast model, $\mathrm{X}=$ number of non-universal & Set-theoretic & Common, Distinctive \\
\hline$\dagger \mathrm{MCM}_{\mathrm{XC}}$ & $\begin{array}{l}\text { features } \\
\text { Common-features distance derived from } \mathrm{MCM}_{\mathrm{X}}\end{array}$ & Set-theoretic & Common \\
\hline$\dagger \mathrm{MCM}_{\mathrm{XD}}$ & Distinctive-features distance derived from $\mathrm{MCM}_{\mathrm{X}}$ & Set-theoretic & Distinctive \\
\hline$\dagger \mathrm{ADCLUS}_{\mathrm{X}}$ & Additive clustering model; $\mathrm{X}=$ number of clusters & Set-theoretic & Common \\
\hline$\dagger \mathrm{DFCLUS}_{\mathrm{X}}$ & $\begin{array}{l}\text { Distinctive-features clustering model; } \mathrm{X}=\text { number of } \\
\text { clusters }\end{array}$ & Set-theoretic & Distinctive \\
\hline$\ddagger \mathrm{GTREE}$ & Additive tree (generalized triples algorithm & Graph-theoretic & Comm., Dis., Uni. \\
\hline$\ddagger \mathrm{GTREE}_{\mathrm{C}}$ & Common-features distance derived from GTREE & Graph-theoretic & Common \\
\hline$\ddagger$ GTREE $_{\mathrm{D}}$ & Distinctive-features distance derived from GTREE & Graph-theoretic & Distinctive \\
\hline$\star$ L2ULTRA & Least-squares ultrametric tree & Graph-theoretic & Common, Distinctive \\
\hline$\star \mathrm{CENM}$ & Centroid metric model (star tree) & Graph-theoretic & Unique \\
\hline$\star$ CENMSQ & CENM fit to squared dissimilarities & Graph-theoretic & Unique \\
\hline
\end{tabular}

Note. $\quad \dagger=$ fit using the Matlab routines available at http://www.socsci.uci.edu/ mdlee/sda.html; $\ddagger=$ fit using the Pascal routines available at http://www.columbia.edu/ jec34/; $\star$ fit using the Matlab routines available at http://cda.psych.uiuc.edu/srpm_mfiles/; $\S=$ fit using the Fortran routines available at 
Table 4

Rank-ordering of non-derived data-collection methods relative to various criteria investigated in this study.

\begin{tabular}{lccc}
\hline & $\begin{array}{c}\text { Dissimilarity } \\
\text { ratings }\end{array}$ & Hierarchical & Free \\
\cline { 2 - 4 } Efficiency & low & medium & high \\
Reliability & high & medium & low \\
Accuracy (group) & high & medium & medium \\
Accuracy (indiv.) & high & medium & low \\
\hline
\end{tabular}

Note. group $=$ group-average data; indiv. $=$ individual data. 


\begin{abstract}
Author Note
Bruno L. Giordano, Mattson Ogg, Bennett K. Smith and Stephen McAdams, Centre for Interdisciplinary Research in Music Media \& Technology (CIRMMT), Schulich School of Music, McGill University, Montréal, Québec, Canada; Catherine Guastavino and Emma Murphy, CIRMMT, School of Information Studies, McGill University. Emma Murphy is currently at the School of Computing of Dublin City University. Portions of this research were reported at the $50^{\text {th }}$ Annual Meeting of the Psychonomic Society in Boston, Massachusetts, USA, in November 2009. This work was supported by Stephen McAdams' Canada Research Chair in Music Perception and Cognition, by a grant from the Natural Sciences and Engineering Research Council of Canada to Stephen McAdams (RGPIN 312774-05) and by a Special Research Opportunity Grant from the Natural Sciences and Engineering Research Council of Canada (NSERC). The authors wish to thank Daniel J. Navarro for discussions on fitting routines for the MCM model.

Correspondence concerning this article should be addressed to Bruno L. Giordano, Schulich School of Music, McGill University, 555 Sherbrooke Street West, Montréal, Québec, Canada H3A 1E3. E-mail: bruno.giordano@music.mcgill.ca.
\end{abstract}




\section{Footnotes}

${ }^{1}$ Hierarchical sorting dissimilarities are more rigorously conceptualized as ordinal measures and should thus be pooled across participants using the median and not the mean. In the present study, the Pearson correlation between median- and mean-pooled hierarchical sorting data is .95 and .97 for the semantic and synthetic sets, respectively. 


\section{Figure Captions}

Figure 1. Estimates of experiment duration as a function of the size of the stimulus set and data-collection method (average stimulus duration $=1 \mathrm{sec}$ ). DR = dissimilarity ratings; $\mathrm{HS}=$ hierarchical sorting; $\mathrm{FS}=$ free sorting; $\mathrm{TS}_{N / 4}$ and $\mathrm{TS}_{5}=$ truncated $\mathrm{HS}$ with a number of starting groups equal to one fourth of the number of stimuli and to five, respectively.

Figure 2. Bootstrap estimates of the reliability $\left(\mathrm{R}^{2}\right)$ of group-average dissimilarities as a function of the number of participants.

Figure 3. Redundancy of dissimilarities collected with different methods. An additive tree (GTREE) is fit to the proportion of variance not shared by data from different methods. The sum of the horizontal tree branches that connect two methods models the amount of variance they do not share. The additive constant has been subtracted from the branch length to improve the metric correspondence between input and tree distances.

$\mathrm{DR}=$ dissimilarity ratings; $\mathrm{HS}=$ hierarchical sorting; FS = free sorting;

$\mathrm{CS}_{X}=$ constrained sorting into $X$ groups.

Figure 4. Redundancy $\left(\mathrm{R}^{2}\right)$ between individual-level free sorting (FS) data and group-average constrained sorts (CS) derived from the hierarchical sorting data, as a function of the absolute difference between the number of CS and FS groups of stimuli. Error bar $= \pm 1$ standard error of the mean.

Figure 5. Redundancy $\left(\mathrm{R}^{2}\right)$ between group-average complete hierarchical sorting (HS) data and derived truncated hierarchical sorts (TS) based on a variable number of starting groups of stimuli. Note that complete hierarchical sorting starts with 20 groups, one for each of the stimuli. 
Figure 6. Left panel: average and standard error of the fit $\left(\mathrm{R}^{2}\right)$ of the distance models across methods and sound sets. Right panel: metric two-dimensional MDS computed on the percentage of variance not shared by different distance models, averaged across data-collection methods and sound sets.

Figure 7. Fit $\left(\mathrm{R}^{2}\right)$ of distance models for dissimilarities from different data-collection methods. Left panel: group-average data; right panel: individual data.

Figure 8. Accuracy of group-average and individual dissimilarities $=\mathrm{R}^{2}$ between dissimilarities and Euclidean distance based on known stimulus features. $\mathrm{R}^{2}$ measures are shown on a logarithmic scale to improve the readability of individual-level results. 


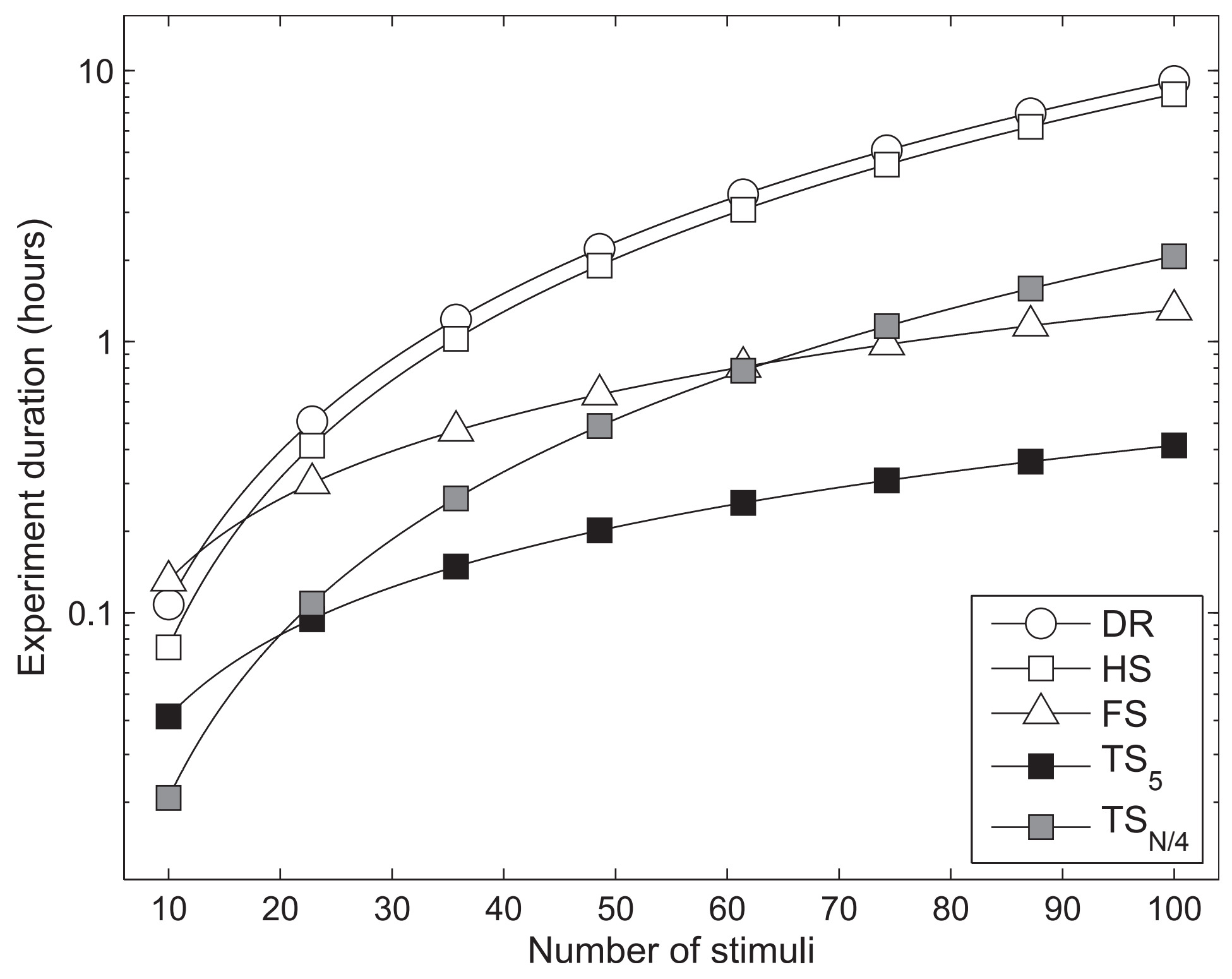




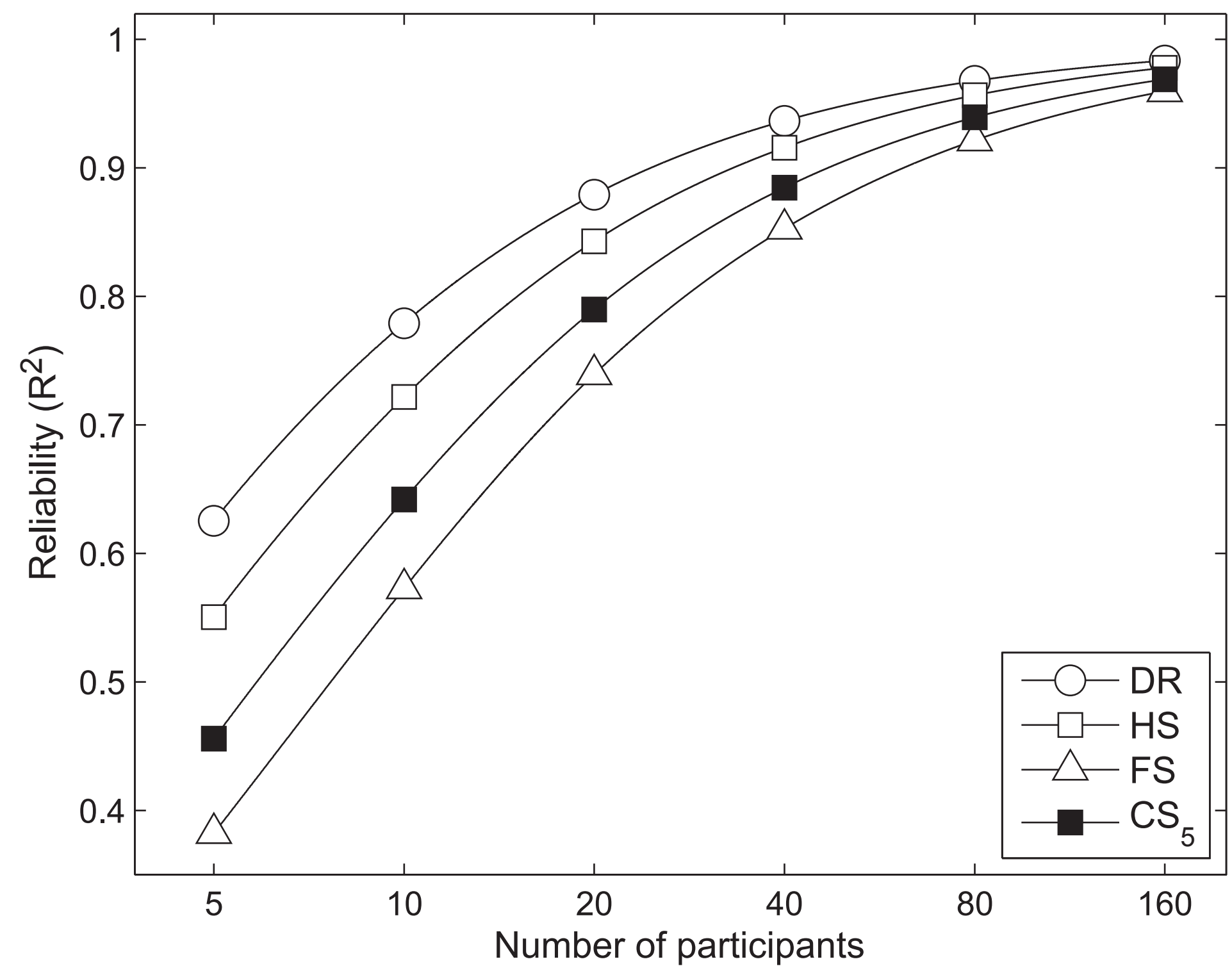




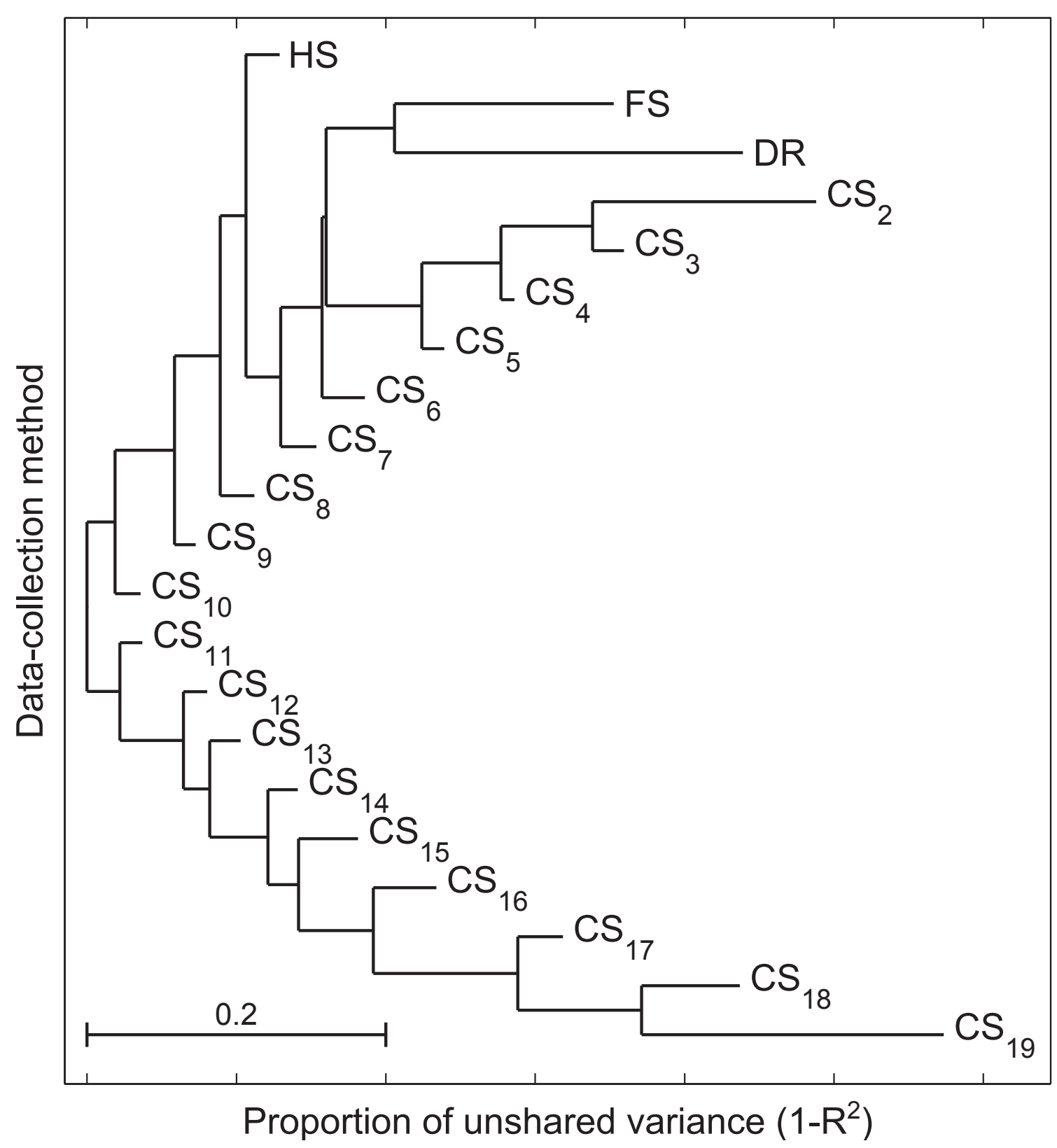




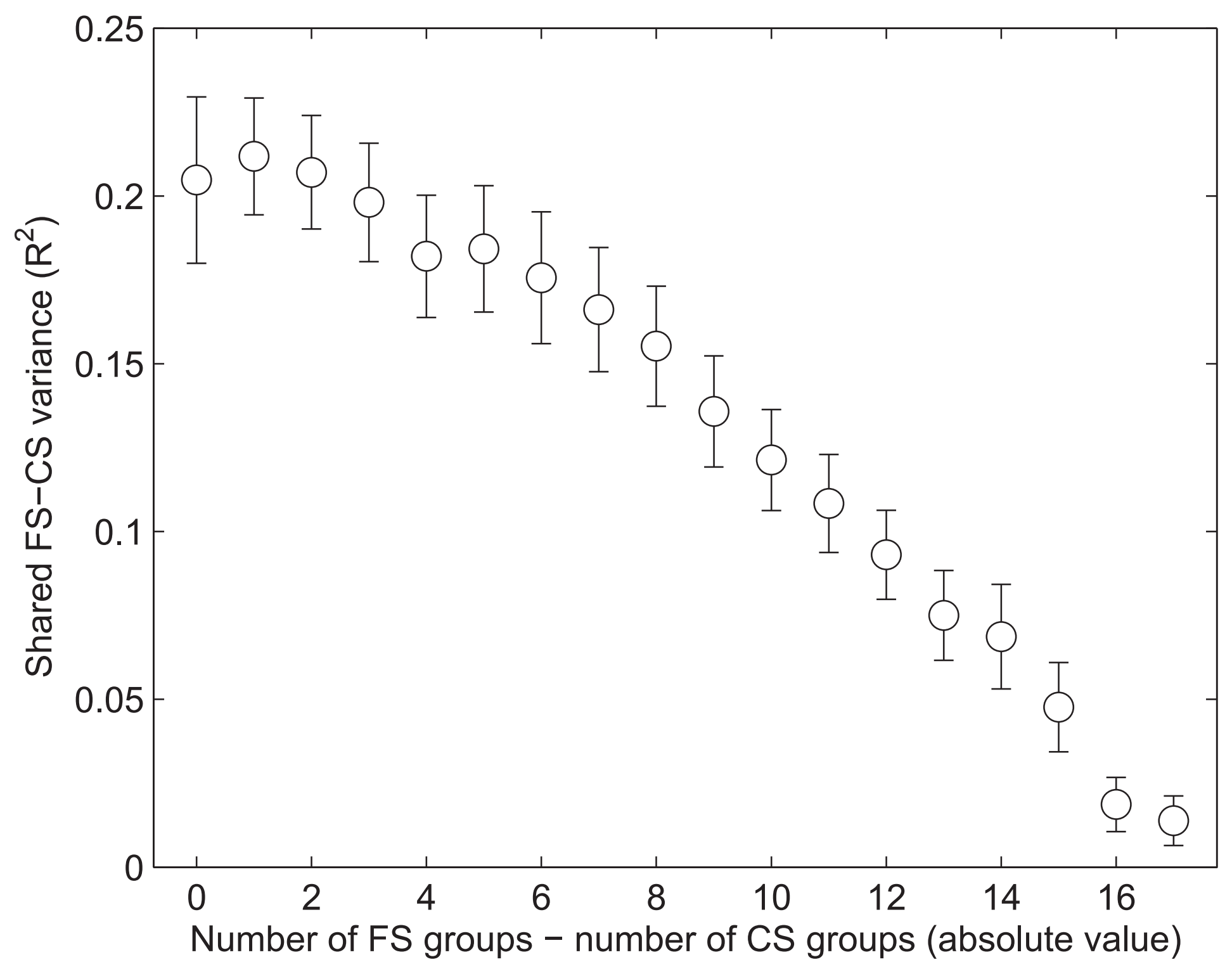




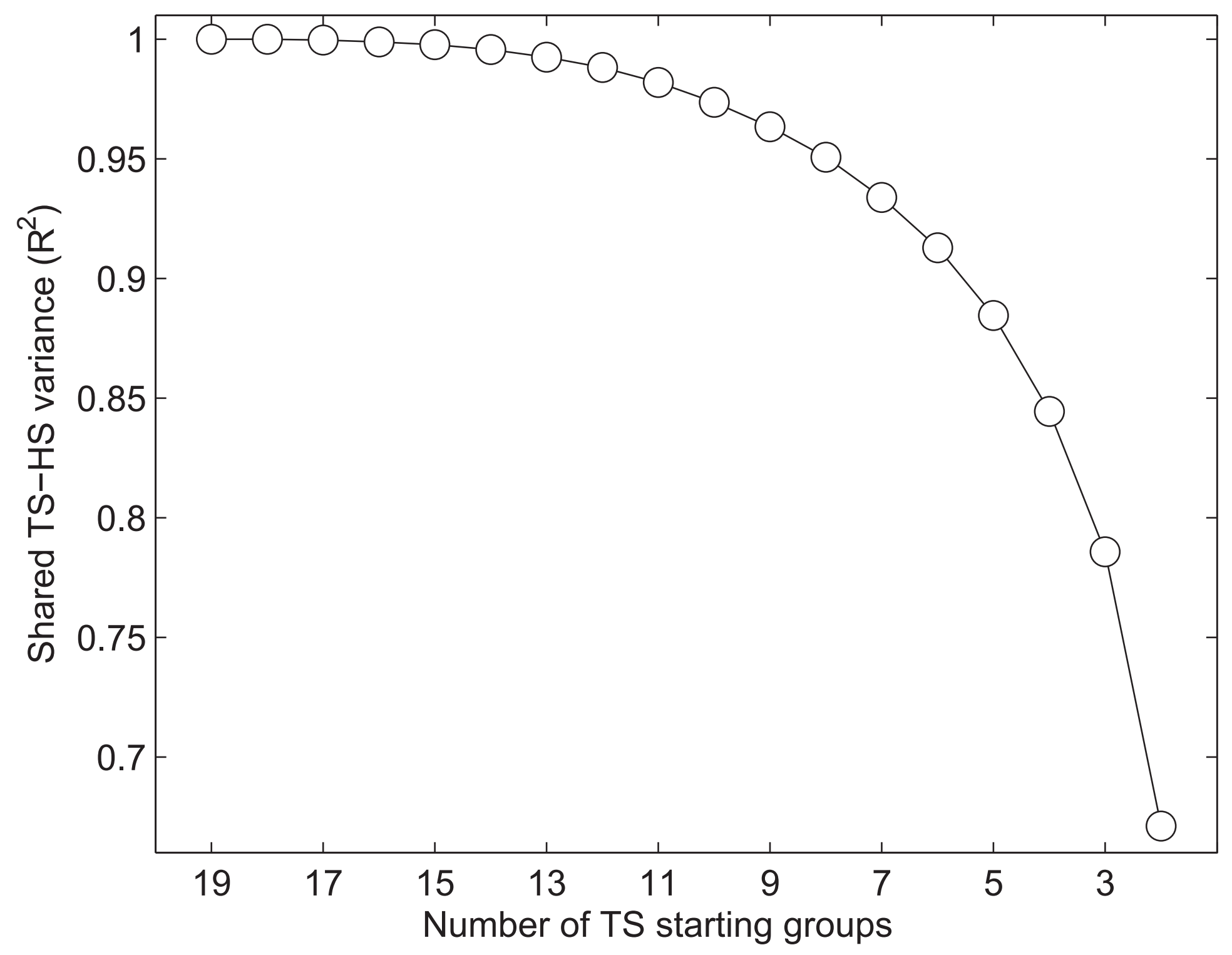




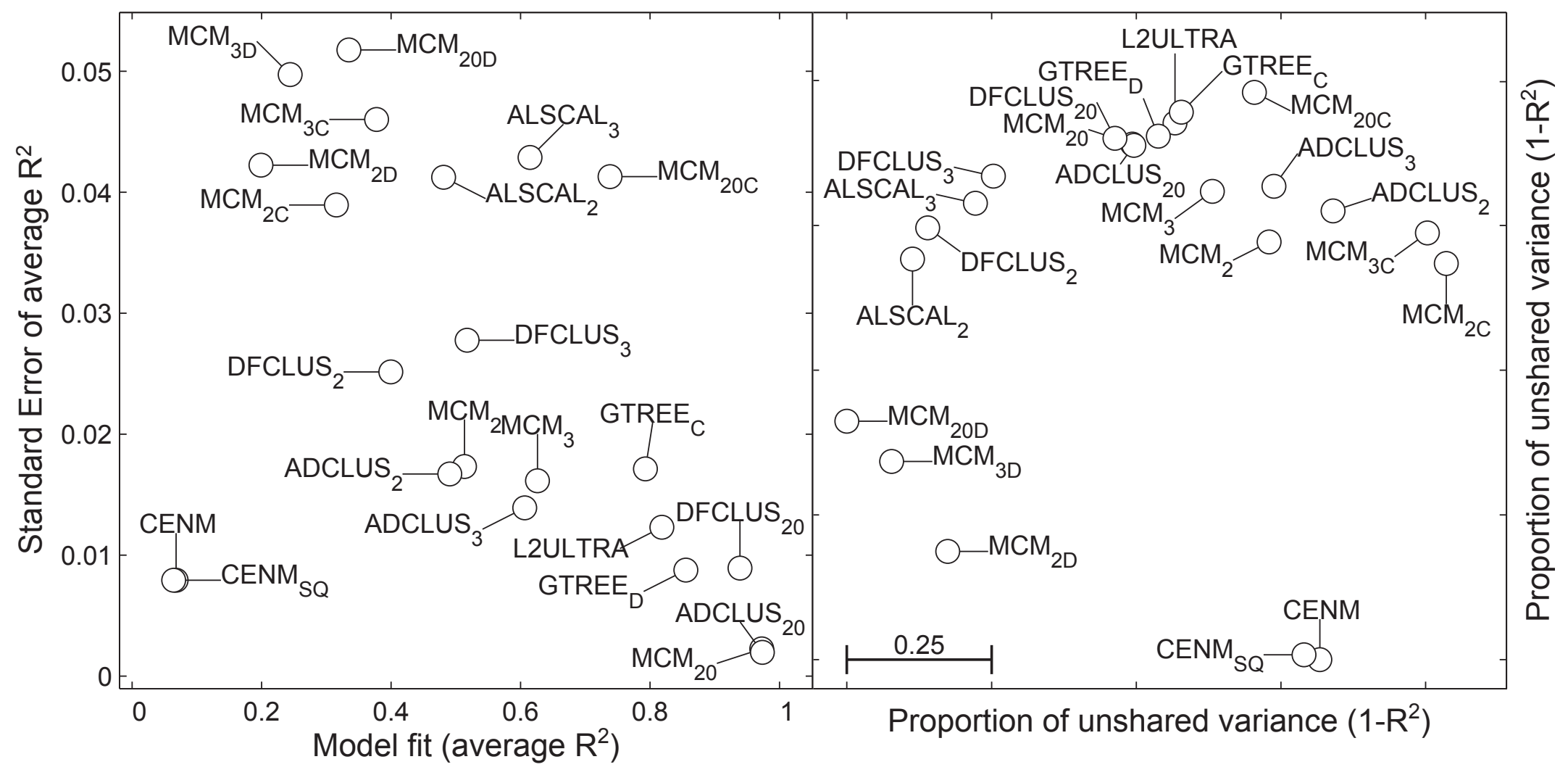




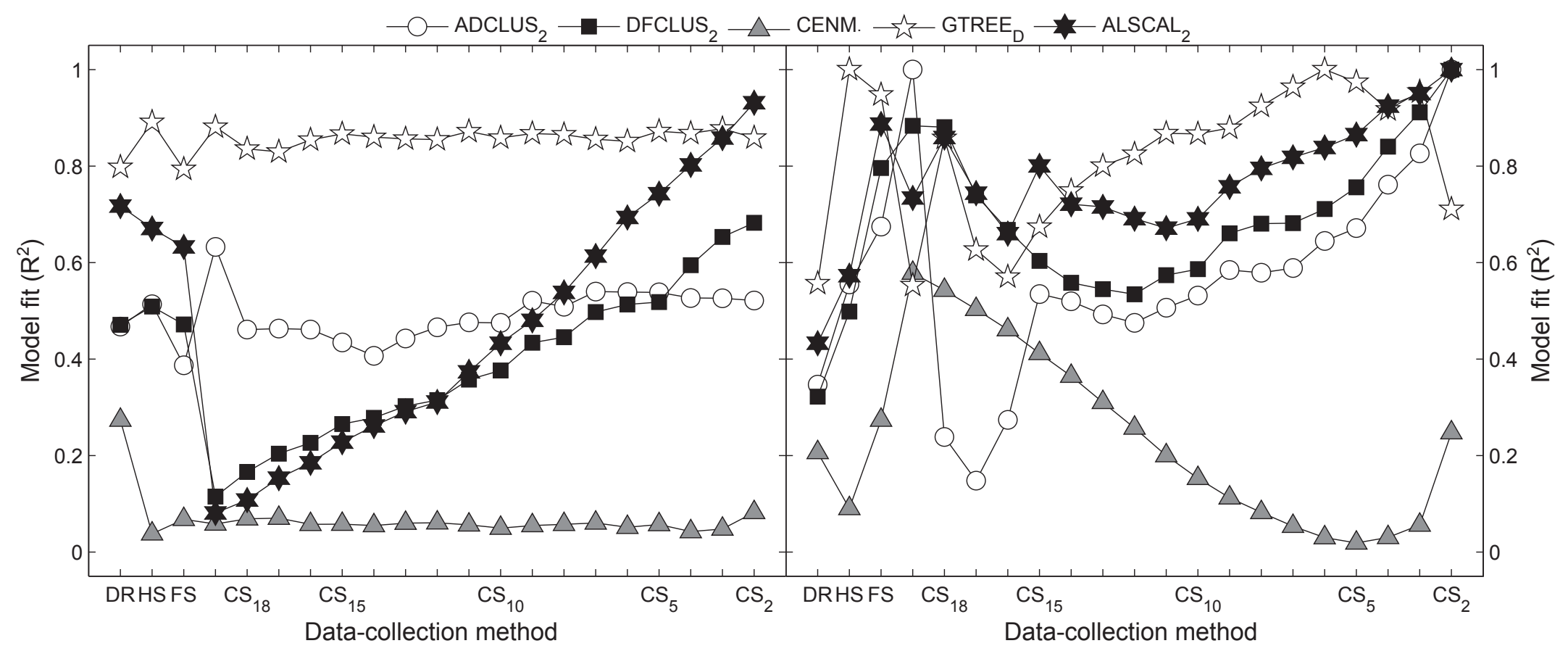




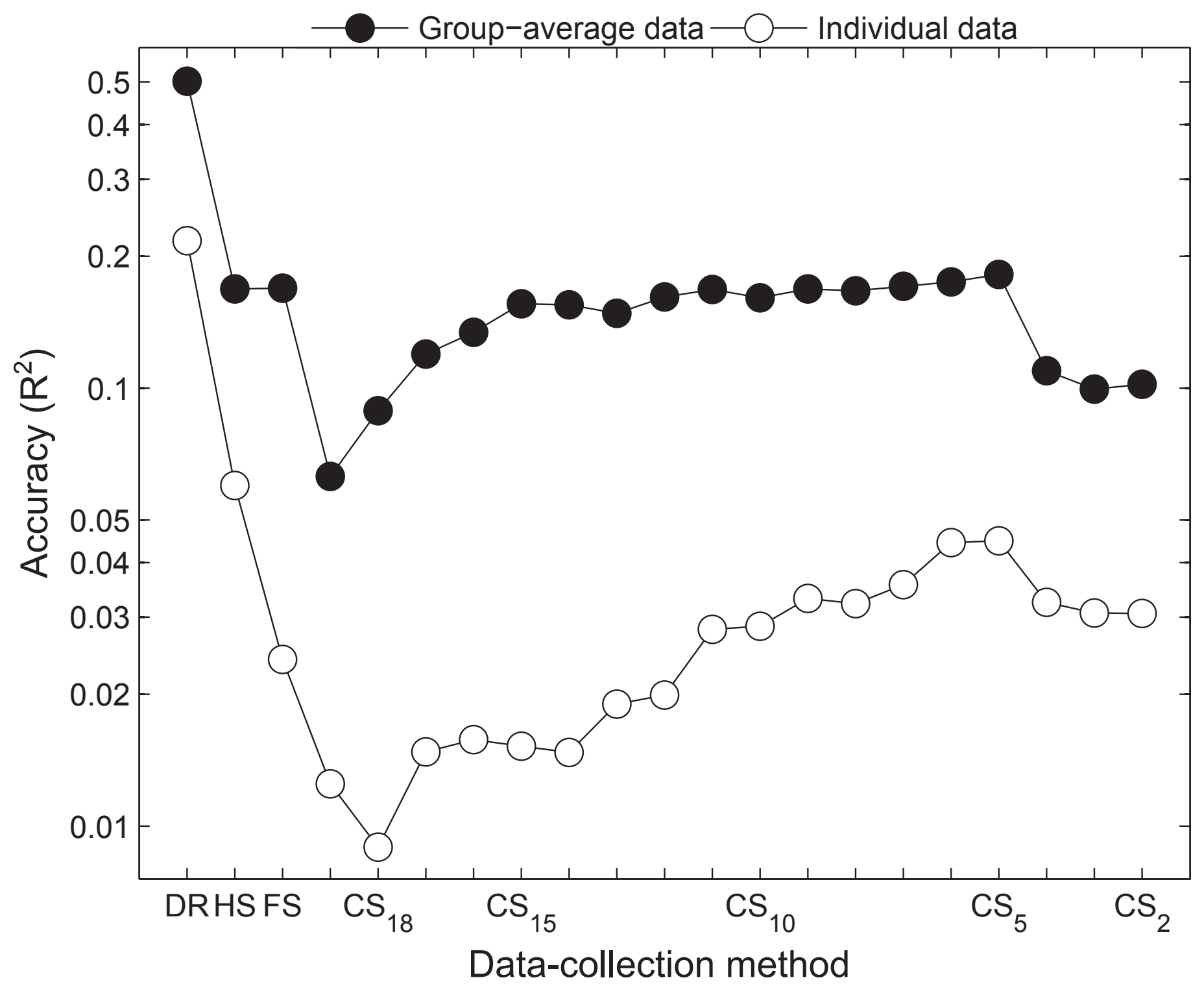

